\title{
Tasting and testing
}

\author{
Inés Crespo ${ }^{1,2} \cdot$ Frank Veltman ${ }^{3,4}$
}

Published online: 10 June 2019

(c) The Author(s) 2019

\begin{abstract}
Our main concern in this paper is the semantics of predicates of personal taste. However, in order to see these predicates in the right perspective, we had to broaden the scope to the wider class of relative gradable adjectives. We present an analysis of the meaning of these adjectives in the framework of update semantics. In this framework the meaning of a sentence is not identified with its truth conditions, but with its (potential) impact on people's intentional states. In this respect, an important characteristic of relative gradable adjectives is the interplay between their evaluative features and people's expectations. The dynamic set-up also makes it possible (a) to model the interpretation of a relative gradable adjective without supposing that the context always supplies a 'cut-off' point determining its application, and (b) to deal in a pragmatic way with situations in which the Sorites paradox arises.
\end{abstract}

Keywords Relative gradable adjectives · Predicates of personal taste · Update semantics · Evaluativity

\section{Introduction}

This paper is about adjectives $G$ with the following properties.

- $G$ can be used in comparative form. We write $G$-er for the comparative form of $G$.

- $G$ has an antonym, $\breve{G}$, which also has a comparative form, $\breve{G}$-er.

$凶$ Frank Veltman

fveltman@mac.com

Inés Crespo

inescrespo@gmail.com

1 NYU Paris, 57, Boulevard Saint-Germain, 75005 Paris, France

2 Institut Jean Nicod, Département d'études cognitives, ENS, EHESS, CNRS, PSL University, UMR 8129. 29, rue d'Ulm, 75005 Paris, France

3 College of Foreign Languages, Hunan University, 2 Lushan South Road, 410082 Changsha, China

4 Institute for Logic, Language and Computation, University of Amsterdam, P.O. Box 94242, 1090 GE Amsterdam, The Netherlands 
- It is logically possible for something to be neither $G$ nor $\breve{G}$-but something 'inbetween'.

- If the object $x$ is $G$, then it is logically possible for there to be an object $y$ that is $G$-er than $x$, and an object $z$ that is less $G$ than $x$. The same holds for $\breve{G}$.

Adjectives with these properties are commonly called relative gradable adjectives (RGA's). Examples are tall with its antonym short, and tasty with its antonym disgusting. ${ }^{1}$

In the following, we will develop a formal account of the meaning of these adjectives, focussing on predicates of personal taste. There is already a gamut of such accounts on the market. Ours differs from all of these in that it does not supply truth conditions, but explains meaning by specifying the changes a statement is intended to induce in the addressee's intentional state. While we hope there is something here for everybody - the contextualist, the relativist, the absolutist, and the expressivist-we also hope to avoid some of the problems that their theories face.

One caveat: This paper marks the start rather than the end of a research project. It contains a lot of hand-waving, and points for further discussion. This is unfortunate, the more so because some of the theories developed in the truth-conditional frameworkin particular the degree-based theories ${ }^{2}$ - offer detailed explanations on issues where we do not yet know what to say. Still, we hope to show that what we say deserves further discussion.

In Sects. 2 and 3 we introduce some concepts, make some distinctions, and lay down some principles that underlie the formal model developed in Sects. 4, 5, and 6. Most of these ingredients are of interest in themselves-independent of the formal modelling.

\section{Context dependence}

\subsection{Comparison bases and comparison classes}

Relative gradable adjectives are called "gradable" because the qualities they describe can vary in intensity - an object can be a little $G$, rather $G$, very $G$. They are called "relative" because the answer to the question whether an object is $G$ heavily depends on context. Consider:

\section{(1) Sue is very talented.}

This can mean many things. It can mean that Sue is very talented at playing the piano, it can mean that Sue is very talented as a manager, it can mean that Sue is very talented in some other area-or just in some areas. And even when it is clear in which area Sue is very talented, we still do not know to whom Sue is to be compared. To everybody his age? To the competitors for the job he is applying for?

\footnotetext{
${ }^{1}$ It is still not clear what the most fruitful classification of adjectives is. A comprehensive discussion of the classification of in particular gradable adjectives can be found in Burnett (2012).

2 Bartsch and Venneman (1972) were the first to present such a theory. Kennedy gave new impetus to the approach. See in particular Kennedy and McNally (2005).
} 


\section{(2) \\ Compared to his classmates, Sue is very talented at playing soccer.}

There are two kinds of context dependence at stake here. To get to grips with them we introduce two notions, the notion of comparison basis, ${ }^{3}$ and the notion of a comparison class.

Every relative gradable adjective $G$ comes with a set $b(G)$ of concepts, here modelled by a set of nouns, each of which establishes a comparison basis for $G$. For example, $b($ skilful $)=\{$ mathematician, violinist, carpenter, $\ldots\}$ or $b($ tall $)=$ \{human being, building, tree\}.

Consider the comparative more skilful than. It is impossible to determine whether or not $x$ is more skilful than $y$ if it is not clear which particular skill is involved. In our terminology, what is needed is a comparison basis. To put it more generally, a concept $N$ establishes a comparison basis for $G$, if it determines a comparative ordering within the domain of objects with the property $N$ which settles for every two objects $x$ and $y$ in the extension of $N$ whether $x$ is a $G$-er $N$ than $y$, or $y$ is a $G$-er $N$ than $x$, or both are equally $G \mathrm{Ns}$.

So, the comparative relation more skilful than with respect to the set of mathematicians may differ from the comparative relation more skilful than with respect to the set of piano players. And it is very well possible that, if two people belong to both sets, one is the better mathematician and the other the better piano player.

If $N$ determines a comparison basis for $G$, then every subset of the extension of $N$ can serve as a comparison class $C$ for $G$. Whether or not an object is $G$ depends not only on $N$ but also on $C$. Whether or not Sue is skilful depends not only on the skill involved-soccer, carpentry, piano playing-but also the peer group. He may be a skilful soccer player compared to his classmates, but that does not mean he is a skilful soccer player compared to all other amateur soccer players.

\subsection{Uniform and pluriform RGA's}

The distinction between comparison bases and comparison classes gives rise to another useful distinction. A relative gradable adjective $G$ is uniform iff every object belongs to at most one comparison basis $N \in b(G)$-where "every" has wide scope over "at most one". If $G$ is not uniform, $G$ is called pluriform. Examples of uniform RGA's are heavy, tall, expensive, and hot; talented and skilful are examples of pluriform RGA's.

Uniform and pluriform RGA's have different logical properties. For uniform RGA's the comparative is intersective; for pluriform RGA's, it is not.

The label intersective is usually only used in connection with the positive form. The positive form $G$ of an adjective is intersective iff the following argument form is valid:

\begin{tabular}{ll} 
premise 1 & $x$ is a $G N$ \\
premise 2 & $x$ is an $M$ \\
\hline therefore & $x$ is a $G M$
\end{tabular}

There are many intersective adjectives, but RGA's are not among them. A short basketball player can still be a tall man.

\footnotetext{
3 We were led to the notion of comparison basis by the discussions on lexical coercion in Asher (2011).
} 
Analogously, we stipulate that for the comparative $G$-er of an adjective to be intersective, the following argument form must be valid:

\begin{tabular}{ll} 
premise 1 & $x$ is a $G$-er $N$ than $y$ \\
premise 2 & $x$ is an $M$ and $y$ is an $M$ \\
\hline therefore & $x$ is a $G$-er $M$ than $y$
\end{tabular}

As the following examples illustrate, this argument form holds for uniform RGA's, but not for pluriform RGA's. ${ }^{4}$

\begin{tabular}{ll} 
premise 1 & Yanjing is a taller basketball player than Fengkui. \\
premise 2 & Yanjing and Fengkui are Chinese men. \\
\hline therefore & Yanjing is a taller Chinese man than Fengkui.
\end{tabular}

premise 1 Yanjing is a more talented mathematician than Fengkui.

premise 2 Yanjing and Fengkui both play the piano.

therefore Yanjing is a more talented piano player than Fengkui. (??)

In English often the preposition for is used to pick out a comparison class, and the preposition as to pick out a comparison basis. ${ }^{5}$ The other way around sounds odd. Compare:

a. For a six-year-old girl she is skilful as a violinist.

b. \#As a six year old girl she is skilful for a violinist.

Of course, there are contexts in which She is skilful for a violinist is fine, but then the skill at stake is not playing the violin, but kick-boxing or something else one would not expect a violinist to be good at.

As is used only in connection with pluriform RGA's. Since for uniform RGA's, for every object only one basis can be at stake, it is odd to suggest that there are more. Compare:

a. Olga is beautiful as a dancer, but not as a singer.

b. \#John is tall as a human being (but not as a ...)

It is strange to use for... when the dots are filled with a phrase referring to a set of objects that do not belong to one and the same comparison basis. Or, as Delia Graff Fara put it, to use for the objects in the comparison class need to be of one kind:

That is why it sounds strange to say that my computer is tall for a thing on my desk, even though it is in fact the tallest thing on my desk. Because the things

\footnotetext{
4 Actually, as long as one stays within one and the same comparison basis also pluriform adjectives are intersective. So if $P$ is a comparison basis, and $M$ and $N$ comparison classes within $P$, the following is valid.
}

\begin{tabular}{ll} 
premise 1 & for an $M x$ is a $G$-er $P$ than $y$ \\
premise 2 & $x$ is an $N$ and $y$ is an $N$ \\
\hline therefore & for an $N x$ is a $G$-er $P$ than $y$
\end{tabular}

5 Recently, the question when to use as and when to use for was raised in Morzycki (2015), who refers back to Siegel (1976). 
on my desk don't form a kind, we have no notion of what a typical height is for a thing on my desk. (Fara 2000, p. 56)

We would put it this way: the things on Fara's desk are incomparable as far as their tallness is concerned, because there is no comparison basis for tall, to which they all belong. Therefore there is no uniform way to compare the things on Fara's desk with respect to their tallness. 6

The distinction between uniform and pluriform RGA's is not to be confused with the distinction between one-dimensional and multi-dimensional adjectives. We would call an RGA multi-dimensional iff the comparative ordering underlying a given comparison basis is composed of several such orderings, each representing one aspect that must be taken into account to determine whether the adjective applies. So for healthy and the comparison basis of human being one has healthy with respect to blood pressure, healthy with respect to lung function, etc. Note, however, that healthy is uniform. An object can be an element of at most one comparison basis for healthy. One cannot be healthy as a human being, but not as a ....

\subsection{Default interpretations}

Consider a sentence of the form $x$ is $G$. For such a sentence to be interpretable, it must be clear what the comparison class and the comparison basis are. In the case of a uniform adjective like tall, often enough information is available about the object $x$ to establish the relevant comparison basis, as in The Eiffel tower is tall. Then the default is to interpret $G$ relative to the comparison class given by this basis.

Consider a sentence of the form $x$ is a $G N$, where $N$ is a noun. In many cases $N$ will define the comparison class. In particular, if $N$ happens to determine a comparison basis for $G$, it seems impossible to interpret $G$ differently. Try to conceive of a situation in which somebody says:

\section{Yanjing is a skilful logician.}

while it is clear from context that the speaker intends to say that Yanjing is a logician who is skilful at playing the piano. That is too far-fetched.

If the noun $N$ does not determine a comparison basis for the adjective $G$, things are different. Consider:

$$
\text { Kyle's car is an expensive } B M W^{8}
$$

Example (6) does not necessarily mean that Kyle's car is expensive for a BMW. That is one reading of the sentence, but aren't all BMW's expensive? Here the speaker

\footnotetext{
6 The things on Fara's desk do not from a comparison class in our sense of the word. In this respect our notion of comparison class differs from the usual. In most of the literature on adjectives, theoretically any set of objects can serve as a comparison class, although in practice the examples dealt with also fit our definition.

${ }^{7}$ Sassoon (2013) contains a thorough discussion (in a degree-based framework) of multi-dimensional adjectives. For the phenomena discussed in the present paper the distinction between one-dimensional and multi-dimensional adjectives is—or seems-not essential.

${ }^{8}$ Cf. Kennedy (2007, p. 11).
} 
may just as well want to say about John's new car that it is expensive for a car and that it is a BMW.

\subsection{For further discussion}

The distinction between comparison basis and comparison class is a fruitful one. We dare say so, although we are well aware that there are still many things to sort out.

One issue that comes to mind is this. As we saw, many adjectives $G$ come with more than one comparison basis. Sometimes this even results in different entries in the lexicon, one for each basis. See for example the lemma for the uniform adjective rich-rich soup, rich man, rich history - in any English dictionary. Now, there must be a reason why we can use the same adjective in all these cases. There must be something that makes us pick more skilful as the the right description for the different comparative relations involved.

On the other hand, different languages make different choices. Consider the adjective tall. In English tall is used both for human beings, trees and buildings. In Dutch, however, you have to use "lang" (=long) in the case of human beings and "hoog" $(=$ high $)$ in the case of trees and buildings. So, the differences between the-somehow related-comparative orderings are sometimes large enough to introduce different adjectives for different bases.

In connection to this: In English one can say Our Christmas tree is taller than you (which is pretty difficult to translate into correct Dutch). This suggests that trees and human beings belong to the same comparison basis for tall. So, the reader ${ }^{9}$ may have wondered why we treated human beings, trees and also buildings as different bases for tall. Why not put the three of them in one basis?

Admittedly, we don't have a watertight story here.

What is lost when we put all these things together in one basis-something like things with height - is the default that when we are talking about some tree, and call it tall, we usually mean that it is tall compared to other trees - and not, say, compared to the building in front of which it is standing. With the huge basis encompassing things with height as a starting point it would be just as natural to pick the building as a comparison as it is to pick the tree standing next to it.

This is one reason why we do not want to give up the idea that human beings, trees and buildings are separate bases for tall. The second reason to stick to this idea has to do with another role that comparison bases play in our story, one that will be extensively discussed below. Roughly, what it amounts to is this: In every comparison basis $N$ there is an important role to play for the set of objects that count as 'normal' in respects relevant to the adjective $G$ concerned. This set is not fixed, it can change over time, but still, every judgment of the form $x$ is a $G N$ is made against a background of what we take, at that point, to be normal.

Now, it may not be very clear what the normal height of a tree would be, or how tall people normally are. Still, it is much clearer in these cases than in the case of things with height. This set of things is so diverse that it makes no sense to look for its normal elements.

\footnotetext{
9 Just as one of our reviewers did, for which we are thankful.
} 
A related question: We claim that it makes little sense to wonder whether $x$ is $G$-er than $y$ independent of a comparison basis. Who is the most talented, John or Peter? It may be that John is more talented than Peter at doing mathematics, and Peter is more talented than John at playing the violin; that one is pretty good at playing soccer, and the other has absolutely no talent for it, etc. Then who is more talented?

And yet, sometimes it does make sense to say something like John is more talented at doing mathematics than Peter is at playing the violin. And Who is more talented? could mean something like Who is more talented, overall? We should be able to analyse the meaning of these sentences without having the comparative more talented than cross the border of any comparison basis. That is not a trivial exercise. (But see Doetjes et al. (2009) for a suggestion how this could be done without introducing scales.)

The correct Dutch translation of The Christmas tree is taller than you runs something like this: "De kerstboom is hoger dan jij lang bent" (something like: "The Christmas tree is higher than you are long"). Once we know how to analyse this without having "hoog" and "lang" cross the border of any comparison basis, we can also analyse the original English sentence as: The Christmas tree is taller (within the basis of trees) than you are tall (within the basis of human beings).

A final question for further investigation: Vagueness comes with levels of granularity. ${ }^{10}$ The comparative orderings of the comparison bases can be more or less refined. For instance, the more we learn and the more refined our palate becomes in wine tasting, the more distinctions we can make. An occasional wine drinker would have no problems comparing Eiswein with Merlot, but an expert would see these as incomparable sorts, and refuse to answer the question which of these is tastier. What is comparable and not comparable may vary; using more refined methods of comparison results in a finer partition in comparison bases. Again, how exactly does this work?

\section{Evaluativity}

\subsection{Weakly versus strongly evaluative adjectives}

Relative gradable adjectives are not very precise. If somebody says $x$ is $G$, one can always ask How $G$ ? And then, in many cases, a more precise characterisation can be given. One can give a rough indication of some man's height, and say that he is tall, give a more precise indication and say a little taller than you are, or even get very precise and say that he is $2.03 \mathrm{~m}$. One can say about some pie that it is tasty, and then get more precise by saying something like but not as tasty as my mother used to make them, or a lot better than the one we had yesterday.

Sometimes there is no need to be precise, ${ }^{11}$ sometimes it is impossible to be precise. ${ }^{12}$ However, these are not the only reasons for using gradable adjectives. Some-

\footnotetext{
10 The notion of granularity turns up at more places in this paper-or it should. It is an important aspect of everything vague, but for the most part we neglect it here.

11 Cf. Fine (1975), for whom vagueness is a matter of nonpedantry: if truth is secured no matter how our words are precisified, it would be pedantic to be more precise.

$12 \mathrm{Cf}$. Dummett (1975), who was the first to point out that vagueness is an essential feature of any language which contains observational predicates.
} 
times we already have a precise qualification, but we want to have a less precise one, too.
Mary: I can run the 3000 meter steeple-chase in $18^{\prime} 57^{\prime \prime}$.
John: Is that fast?

Why would John want to have an imprecise qualification if he already knows exactly how fast Mary is?

\section{Mary: I can run the 100 meter sprint in 12 seconds.}

John: Wow, that's fast!

Relative gradable adjectives are not only imprecise, we also use them evaluatively. This becomes particularly clear from their role in exclamatives. Compare:
a. What a delicious desert you prepared!
b. How tall you are!
c. \#How 2.03 m you are!
d. \#How digital this watch is!

One cannot use measure phrases or non-gradable adjectives in an exclamation. There must be something gradable there.

Some RGA's are, in a way, more evaluative than others. Compare tasty and tall. The difference is best explained by looking at their comparatives.
a. Yanjing is taller than Fengkui.
b. This cake is tastier than that pie.

The question who is taller, Yanjing or Fengkui, is a factual question; there exists a public procedure settling it. However, there is no public procedure settling the question which is tastier, the cake or the pie. The comparative tastier than is no less subjective than the positive tasty.

Many RGA's behave like tall. We will call them weakly evaluative to distinguish them from the RGA's behaving like tasty, that we will call strongly evaluative. ${ }^{13}$ All adjectives called predicates of personal taste in the literature belong to this class.

We must be more precise. It is not so much the adjective that is weak or strong, but the adjective with respect to a given comparison basis. The possibility is not excluded that the adjective $G$ in relation with one basis comes with a public procedure settling

\footnotetext{
13 A similar observation is made in Kennedy (2013), who notes that while you can get faultless disagreements with examples like (10-b), you don't get faultless disagreements in cases like (10-a). Kennedy explains this on the basis of the dimensional vs evaluative distinction made by Bierwisch (1989).

The reader might wonder whether the distinction we are making is the same, with Bierwisch's dimensional adjectives covering our weak evaluatives and his evaluatives corresponding to our strong evaluatives. Our distinction is inspired by Bierwisch's, but it is different in two respects. First, for Bierwisch dimensional adjectives like fast or heavy are ambiguous or polysemous, having one lexical entry associated with an objective scale and another one that is evaluative. We think it is counterproductive to postulate a case of polysemy here, because this leaves unexplained the relation between evaluative and non-evaluative uses of RGA's. Second, some weak evaluatives, e.g., soft or hard, are not associated with an objective scale along some dimension but nonetheless have a public procedure settling the question whether $x$ is softer/harder than $y$. For us these are weakly evaluative, but Bierwisch has to classify them as evaluatives.
} 
questions of which objects are $G$-er than which other objects, whereas for some other basis there is no such public procedure. Consider the adjective tall. This adjective is weakly evaluative with respect to the comparison basis consisting of buildings, and strongly evaluative with respect to the comparison basis consisting of stories.

Weak and strong evaluatives behave differently, semantically and pragmatically. This becomes clear when we take a closer look at evaluative disagreements.

Suppose that Mary claims that Yanjing is tall, but John disagrees. Suppose that both are looking at Yanjing, so they can see how tall he is. Suppose on top of this that for both of them the relevant comparison class is the class of Chinese men. This is an example of a purely evaluative disagreement. There is no disagreement about the facts; both will indicate the same height with their hands when they state: Yanjing is $\underline{\text { this }}$ tall. But Mary claims that this is tall for a Chinese man, and John claims it's not. ${ }^{14}$

In these circumstances, a typical reaction to Mary's claim would be for John to say: No, this height is quite normal for a Chinese man. Then Mary might say something like: No, no, you are wrong, the average height of adult Chinese men is somewhere between $1.65 \mathrm{~m}$ and $1.70 \mathrm{~m}$, and Yanjing is at least $1.75 \mathrm{~m}$.

That is how the discussion proceeds in many, if not all, cases where the question at stake is whether $x$ is $G$ for some weak evaluative $G$. To a child who thinks (s)he gets too little pocket money, we say: No, no, 5 EUR is quite normal for a child your age, ask your classmates. To someone who wonders how fast it is to run the 3000 meter steeple-chase in $18^{\prime} 57^{\prime \prime}$, we can say that this is very slow because normally people who run this track finish within 10 minutes.

More generally, given a comparison class $C$ and comparison basis $N$, the question whether $x$ is $G$ reduces to the question whether $x$ is $G$-er than elements of $C$ normally are. And the crux is that for weakly evaluative RGA's, as soon as the contestants agree on what counts as normal, this question reduces to a purely factual question.

In the case of strong evaluatives, disagreements are much more complex. It is not difficult to see why. Suppose that Mary and John have both tasted a piece of the same cake. Mary claims that the cake is tasty, John claims it's not. Assume that for both of them the comparison class is the class of pastries. And suppose-just for the sake of argument - they even agree on a standard of tastiness: every pastry at least as tasty as the apple pie they had last week, counts as tasty. Now, the difference with the disagreements we discussed above is that the question at issue has not been reduced to a question of fact. Mary can find the cake tastier than the apple pie they had last week and John can find the opposite, and there is no public procedure to decide who is right.

This holds for all strong evaluatives: Settling a disagreement about the question whether $x$ is $G$ is not just a matter of agreeing on a standard. Trying to agree on a standard only makes sense if the underlying comparative ordering is the same for all parties. But in the case of strong evaluatives this rarely happens. ${ }^{15}$

\footnotetext{
14 Barker (2002) calls this a "metalinguistic mode of use".

15 In this respect, the theory presented in Barker (2013) works well for weak evaluatives, but not for strong evaluatives.
} 


\subsection{The normality principle}

We said that in case of disagreement about the applicability of a weak evaluative the following holds: As soon as the contestants agree on what counts as normal, the question whether $x$ is $G$ reduces to a purely factual question.

Now, this does not mean that whenever two people give the same answer to the question whether $x$ is $G$, they agree on what counts as normal. It is very well possible that for each of them $x$ is $G$-er than any normal object, while for each of them what counts as normal is different. Only when the one thinks that $x$ is $G$ and the other that $x$ is not $G$, a discussion about what is normal may arise, and even then they can often come to agree without sorting out whether what the one considers normal exactly coincides with what the other considers normal.

Of course, sometimes the discussion will not stop before an exact standard has been set. Nobody wants to buy a car that is too expensive, and nobody wants to sell it too cheap. So, what is a normal price for a used BMW with 150k miles on it - not expensive, not cheap, but exactly right? (In such cases there can be no 'something in-between', one has to draw the line somewhere, often rather arbitrarily just to put an end to the discussion.)

For weakly evaluative $G$ 's, the following principle holds. If something is normal in the respects relevant to $G$, then it is neither $G$ nor $\breve{G}$. This is not a quirk of nature, this is how language works. If something is normal, it is in line with our expectations, and therefore not worth mentioning. We use weak evaluatives and their antonyms to signal something special, unexpected, and therefore worth mentioning. That's how their evaluative effect comes about. ${ }^{16}$

"Normal" is not the same as "average". Compare:

a. Nadia's height is greater than the average height of a gymnast, but she is still not tall for a gymnast.

b. \# Nadia's height is greater than normal for a gymnast, but she is still not tall for a gymnast.

Example (11-a) is taken from Kennedy (2007). Clearly, it is not a contradiction. Kennedy concludes from this that the cut-off point for being $G$ in the comparison class $C$ is not supplied by the average degree of $G$-ness in this class. Rather, the cutoff point should be chosen in such a way that it distinguishes objects that stand out in the context $C$ relative to a measure of $G$-ness. ${ }^{17}$ For us, these are just the objects that are $G$-er than normal. Note, however, that normal does not define to a cut-off point. The range of normal is, indeed, a range, and its borders are indeterminate.

It is normal to be neither $G$ nor $\breve{G}$, but not the other way around. There are comparison classes that are so accidental that there is no normality to discover. Consider the children in an arbitrary primary school class. There is an average height there, and

\footnotetext{
16 Expectations do not only play an important role in the interpretation of RGA's, but also in the interpretation of quantifiers like many, and few, generic sentences, and subjunctive conditionals. For many, things started with Fernando and Kamp (1996) and Égré and Cova (2015) shows that both descriptive and normative expectations play a role. For the other topics, see Crespo et al. (2018).

17 Cf. Kennedy (2007, p. 20).
} 
the children with this height will count as neither tall nor short. But there is no such thing as a normal height there (although the children themselves may think there is).

\subsection{The principle EOI}

Relative gradable adjectives give rise to the Sorites paradox. This holds in particular for the ones whose applicability is decided directly by sensory perception, since their use is guided by what Hans Kamp coined the Principle of Equivalence of Observationally Indistinguishable Objects (EOI). ${ }^{18}$

If two objects are observationally indistinguishable in the respects relevant to $G$, then either both satisfy $G$ or else neither of them does.

"Observationally" is actually too restricted a term, since we are interested in all sorts of perception, not only visual perception.

With such an adjective $G$, one can always find a comparison basis $N$ with the following property: There are objects $d$ and $e$ in the extension of $N$ such that:

a. $G$ clearly applies to $d$;

b. $G$ clearly does not apply to $e$;

c. There is a sequence of objects $d=d_{0}, d_{1}, \ldots, d_{n-1}, d_{n}=e$ in the extension of $N$ such that for no successive objects $d_{i}$ and $d_{i+1}$ in this sequence, $d_{i}$ is observationally $G$-er than $d_{i+1}$, or $d_{i+1}$ is observationally $G$-er than $d_{i} .{ }^{19}$

Let us write $x \approx_{G, N} y$ iff with respect to the comparison basis $N$ neither $x$ is observationally $G$-er than $y$, nor $y$ is observationally $G$-er than $x$. Whenever $x \approx_{G, N} y, x$ and $y$ are observationally indistinguishable in the respects relevant to the adjective $G$, and therefore it is tempting to read $x \approx_{G, N} y$ as $x$ is just as $G$ as $y$. We will not resist this temptation, even though $\approx_{G, N}$ need not be an equivalence relation. That is, $\approx_{G, N}$ is reflexive and symmetric, but generally not transitive. It may very well occur that there is no observational difference between the objects $x$ and $y-x$ is detectably just as $G$ as $y$-and no discriminable difference between the objects $y$ and $z-y$ is detectably just as $G$ as $z$-whereas $x$ and $z$ can be discriminated- $x$ is detectably $G$-er than $z$.

It is easy to see now how the paradox can arise. The Principle EOI forces us to use the adjective $G$ in a way that would only be coherent if the relation $\approx_{G}$ were transitive. For, what else does this principle express but:

For any $x, y$, if $x \approx_{G, N} y$ and $G$ applies to $x$, then $G$ applies to $y$.

So, you cannot assign the adjective $G$ to $d_{0}$ without having to assign it to $d_{1}, d_{2}, d_{3}, \ldots$, and, finally, to $d_{n}$ as well. EOI forces you to do so, even though $\approx_{G, N}$ is not transitive: $d_{0}$ and $d_{n}$ could be so far apart- $d_{0}$ is $160 \mathrm{~cm} d_{n}$ is $210 \mathrm{~cm}$, the temperature of $d_{0}$ is $2^{\circ} \mathrm{C}$, the temperature of $d_{n}$ is $80^{\circ} \mathrm{C}$ - that it would seem perfectly alright to say that $d_{0}$ is $G$-say short, or cold-but that $d_{n}$ is not.

It looks like we are in a predicament: either we try to modify the Principle EOI so that no paradox can arise, or we stick to it, in which case it looks like we must learn to live with the fact that the rules governing our way of speaking sometimes lead to paradox.

18 See Kamp (1981). What follows is also very much inspired by Dummett (1975).

19 Kamp (1981) sketches a similar scenario. 
Most logicians choose the first horn of this dilemma. They want to get rid of the paradox. But there is a price to pay. If we were to follow their advice, we should be much more careful using RGA's than the Principle EOI allows. But why would we do so? Even if the sloppy way we use these adjectives sometimes leads to paradox, we might still refuse to change this way of using them because in the vast majority of cases this sloppy way serves its purpose just fine. This is the reaction a philosopher like Wittgenstein would have to any solution that proposes to modify the EOI Principle. In the Philosophical Investigations Wittgenstein compares meaning rules with signposts and he writes:

The signpost is in order-if, under normal circumstances, it fulfills its purpose.

(Wittgenstein 1958, §87)

In other words, there is nothing wrong with the Principle EOI. After all, normally we are dealing with just a few objects, most of them very well distinguishable from each other. In those circumstances EOI does not give rise to inconsistency; normally, it serves its purpose quite well. Only in exceptional situations-i.e., when we are confronted with sequences of objects as described above-things go wrong. But then, RGA's like cold and tall are not meant to be used in those situations; what we should use there is other, finer tools; we should no longer talk in terms of cold, and short, etc., but in terms of degrees Celsius or millimeters. The Sorites paradox typically arises when the coarse tools of vague adjectives are used side by side with these finer tools, i.e., when one starts saying things like: If someone with height $190 \mathrm{~cm}$ is tall, then so is someone who is $189.9 \mathrm{~cm}$. As such, it merely reflects that one should not use the coarse tools in circumstances where only other, more delicate, ones are applicable. ${ }^{20}$

We will stick to EOI. Consequently, according to the semantics we will sketch in the next section, situations may arise in which the use of RGA's leads to paradox. We don't care, because there are lots of situations left in which they work well-just like they do in real life.

Observational indistinguishability is just one of the sources generating Sorites-like situations. Another is semantic indeterminacy. ${ }^{21}$ Example: consider the adjective tall, and a comparison class consisting of 50 adult men of length $151 \mathrm{~cm}, 152 \mathrm{~cm}, \ldots$, and $200 \mathrm{~cm}$ respectively. So, there is no observational indistinguishabilty here. Still, where would you draw the line between tall and not tall? And wherever you draw it, why draw it right there and not one or two centimeters higher or lower? And wouldn't you agree that if somebody of length $n \mathrm{~cm}$ is tall than so is somebody of lenght $n-1 \mathrm{~cm}$ ? So, then why aren't all these men tall?

This situation is less problematic than the one sketched in connection with the Principle EOI. If you draw a line somewhere-even if you do so arbitrarily-, once it is drawn it is clear who counts as tall and who does not. But in the earlier case, if you pick a particular object $d$ and proclaim that everything at least as $G$ than this $d$ is $G$, you still cannot determine which objects are $G$ - not by the observational means at hand, that is.

\footnotetext{
20 This point is extensively discussed in Weiss (1976).

21 See Égré (2015) for an in-depth discussion of this notion and a comparision with the notion of observational indistinguishability (which he calls "epistemic indiscriminability").
} 
We do draw lines, sometimes we have to draw a line somewhere even though it can only be drawn arbitrarily - think of a teacher grading exams and having to decide which score counts as satisfactory, or of a legislator having to decide which speed is still safe. Why set it at $120 \mathrm{~km} / \mathrm{h}$, say, and not at $125 \mathrm{~km} / \mathrm{h}$ ?

We are well aware that the above is at best the first section of a more thorough discussion of what we would like to call a pragmatic - or maybe better practicalsolution to the Sorites paradox, in particular because this paradox has many forms, and not all of them will have the same (re)solution. ${ }^{22}$ Here, we have only dealt with the simplest form, where the premises bring you from a sentence of the form $G a_{0}$ via 1000 applications of $E O I$ to $G a_{1000}$, whereas for independent reasons you also accept $\neg G a_{1000}$. For this case there is no way out. Dummett ${ }^{23}$ concludes that this shows that Frege was right when he claimed that natural language, or at least the use of vague expressions, is fundamentally incoherent. We would like to qualify this and add something like: in some-rare-circumstances.

\subsection{The experience principle}

It is distinctive of relative gradable adjectives that they are felicitous in embeddings under the attitude verb find.
a. I find this car expensive.
b. I find this cake tasty.
c. \#I find the number 20 even. ${ }^{24}$

For our own ease, we will call sentences like (12-a) and (12-b) restricted judgments, in contrast to unrestricted judgments like This car is expensive or This cake is tasty.

This section is concerned with the differences in meaning between these two types of expressions. One way in which they differ is illustrated by the following examples:

a. This brand of coffee used to be tasty, but it isn't any more.

b. I found this brand of coffee tasty, but I don't anymore.

When you stop liking a certain brand of coffee, this can be the result of two different sorts of changes: something in the coffee - the beans, the roasting process, etc.- has changed, or you-your 'taste' for coffee-changed. You can assert (13-b) in both circumstances, but if you think it is just your appreciation that changed, it would be misleading to assert (13-a). Apparently, even though tasty is strongly evaluative, we think of the coffee's tastiness as something inherent in the coffee.

Something similar can be said about other strong evaluatives. Compare:

a. I used to find Mozart's Requiem beautiful, but I don't any more.

b. Mozart's Requiem was beautiful, but it isn't any more. (??)

\footnotetext{
22 See Kamp and Sassoon (2017) for a thorough inventory of the problems one has to deal with.

23 Dummett (1975, p. 319).

24 Sæbø (2009) was the first to note that only gradable adjectives are felicitous under find. According to him, the complement of a find construction should feature a judge argument. Our approach, like Pearson (2013), proposes a judge-free account of the contrast in Example (12).
} 
Given that Mozart's Requiem has not changed, how can its beauty have changed?

Much of what we say in this section is inspired by Kant's account ${ }^{25}$ in the third Critique $^{26}$ of what he called taste judgments ("Geschmackurteile"). ${ }^{27}$

One of the things he says is in line with the above: When we say This landscape is beautiful, we speak "as if beauty were a property [Beschaffenheit] of the object and the judgment logical (constituting a cognition of the object through concepts of it)." 28 But he also says that taste judgments are grounded on feeling, and in particular on a feeling of pleasure or displeasure.

This brings us to the second difference between I find this $G$ and This is G. In most, if not all, contexts This is $G$ implies I find this $G$.
a. This Picasso is beautiful,...
b. \# ...I don't find it beautiful.
c. ...she doesn't find it beautiful.

There is a remarkable asymmetry here between the first- and third-person restricted judgment. You cannot accept (15-a) if you accept (15-b), but there is nothing wrong in continuing (15-a) with (15-c). ${ }^{29}$

The conflict between (15-a) and (15-b) disappears if (15-a) is replaced by a generalisation of a restricted judgment, as in Almost everybody finds this Picasso beautiful, or in People normally find this Picasso beautiful. So, somebody who asserts This is beautiful is not just reporting the results of a survey.

Similar remarks can be made about other strong evaluatives. You cannot sincerely say that a particular dish is tasty if you have never tasted it, or that a particular match was boring if you have not watched it. In such cases you can say something like Presumably this dish is tasty, or The match must have been boring, but you cannot assert the unrestricted judgments. ${ }^{30}$

\footnotetext{
25 We are not the first to think that Kant's work on this topic is important for semantics. Both Umbach (2016) and Buekens (2011) refer to him.

26 Kant (1790). References below correspond to paragraphs in Part One, Analytic of aesthetic judgment. Book I, Analytic of the beautiful.

27 One might be inclined to think that on Kant's account This cake is tasty is a paradigmatic example of such a Geschmackurteil, but it is not. Kant would classify this sentence as a judgment of the agreeable, whereas for him an aesthetic judgment like This landscape is beautiful is paradigmatic for a taste judgment. Both kinds of judgment are grounded on feeling, but according to Kant judgments of the agreeable do not make a claim to the agreement of others, whereas taste judgments do. We think, pace Kant, that both do. If one follows Kant in making it essential to the meaning of adjectives like tasty that they have mere private validity, it becomes difficult to explain the difference between I find this cake tasty and This cake is tasty. Moreover, Kant's dismissal of the normativity of judgments of the agreeable makes it difficult to understand why there would ever rise a disagreements about gustatory matters. Further reasons not to follow Kant too closely are developed in Crespo (2015).

28 Kant $(1790, \S 6)$.

29 Pearson (2013) makes the same point, using a similar example. See also Moltmann (2010) and Ninan (2014).

30 As Pearson (2013) puts it: "If I have good reason to believe that shortbread is tasty, say because a reliable expert has told me so, I might say, Apparently, shortbread is tasty, but not, Shortbread is tasty." We very much agree with her observations.
} 


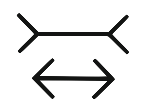

Fig. 1 The Müller-Lyer illusion

To put it more generally, for $G$ strongly evaluative, in most, if not all contexts, one cannot accept This is $G$ if one does not accept I find this $G$. We call this the Experience Principle. ${ }^{31}$

The Experience Principle does not extend to weak evaluatives. You need not have lifted a suitcase yourself to sincerely say that it is heavy if for instance you know its weight as measured by a scale. Or, to give another example, when looking at Fig. 1, you can sincerely say:

\section{I find the upper arrow longer than the lower one, but (in fact) it isn't.}

No major conflict arises here, although a certain awkwardness is felt, a mismatch between experience and facts. Note also that although your belief that the two segments are of equal length might be wrong, there is a sense in which you cannot be mistaken about how they appear to you. More generally, our experience is in a sense incontrovertible: I may be wrong about the question whether what I am eating is a cake or a pie, I may lie and say that I like it, while I find it bland, but I cannot be wrong about my appreciation of it. In the case of the Müller-Lyer arrows, when I realise that the two segments are in fact equally long, I may still say: I find the upper arrow longer than the lower one.

One question remains: can there be a situation analogous to the Müller- Lyer involving a strongly evaluative RGA rather than a weakly evaluative one? Given what we say below, we are inclined to think this is not possible.

\subsection{The universality principle}

For strongly evaluative RGA's, a third difference between This is $G$ and I find this $G$ is this: This is $G$ comes with expectations of about what other people should find. Other people are expected to find this $G$ as well. We call this the Universality Principle. This, too, is in line with Kant's ideas,

...Whether a garment, a house, a flower is beautiful: no one allows himself to be talked into his judgment about that by means of any grounds or fundamental principles. One wants to submit the object to his own eyes, just as if his satisfaction depended on sensation; and yet, if one then calls the object beautiful,

\footnotetext{
31 We are not the first to say so. In addition to Pearson (see the footnote above), Ninan (2014), and MacFarlane (2014) make similar observations. Ninan uses the phrase "acquaintance inference" for what we call the Experience Principle. We want to avoid the term "acquaintance" here as it suggests that Russell's distinction between knowledge by acquaintance and knowledge by description has anything to do with it. MacFarlane introduces tastes, to explain what is going on. These tastes act like private systems of measurement, the idea being that a taste determines a threshold for an individual. "One's tastes, too, serve as a gustatory standard, quite independently of whether one can articulate this standard." Towards the end of Sect. 3.4 we will discuss some problems we have with this kind of view.
} 
one believes oneself to have a universal voice, and lays claim to the consent of everyone... (Kant $1790, \S 8$ )

Two remarks are in order. Firstly, it will make a huge difference to your actions if you think that something is tasty or beautiful and you not just find it so. Our actions (linguistic and non-linguistic) are for a large part determined by our expectations. If I think that some dish $i$ s tasty, and therefore expect you to find it tasty as well, I may consider preparing it for our dinner. If I think it is just me who finds it tasty, I will probably not consider it.

Secondly, the expectations involved in the Universality Principle have both an epistemic and a normative side. If I believe that something is tasty or beautiful, you must find it tasty or beautiful as well—and here "must" does not only mean that I will presume that you will find it tasty or beautiful (unless I know you don't), but also that I think you should ${ }^{32}$ find it tasty or beautiful (even if I know you don't). If you don't, I will try to change your mind-or rather your taste. ${ }^{33}$

\subsection{An amendment}

Where we wrote "Other people are expected to find this $G$ as well", obviously, "People" has to be qualified. We do not expect children to find cognac tasty.

But this is not the only exception we have to make. Normally, speaker and addressee are forming expectations about a community to which both of them belong. That is when the Universality Principle, and also the Experience Principle fully apply. But not all conversations are like that. Take for example the case of a teacher addressing her students:

\section{This exercise is difficult.}

The teacher may say so, even though she herself does not find the exercise difficult. Or consider the next example, due to Kai von Fintel, and discussed in Stephenson (2007):

Mary: How's that new brand of cat food you bought?

Sam: I think it's tasty, because the cat has eaten a lot of it.

\footnotetext{
32 One of our reviewers notes that not all judgments concerning taste make claims of the same strength, let alone they make claims of the same strength as moral judgments and writes: "It does not strike me as totally obvious that a judgment like: Paul from Massage Envy gives pleasant massages has the same claim of universality as does: Waterboarding is morally wrong." Admittedly, there is a difference in strength here, but we are not sure the difference is to be found in a difference in how the Universality Principle applies. If it is, our view could incorporate this because it allows for a variable $O$ representing the community. But we're not sure that fully explains the difference.

33 There is some discussion among interpreters about whether in Kant taste judgments are normative or merely predictive of how fellow cognitive agents should or will respond to a given object. For instance, Guyer (1979, pp. 139-147 and pp. 162-164) thinks that Kant's taste judgments just set rational expectations about others' perceptual states, while Ginsborg (1990) and subsequent work argues that taste judgments are normative because Kant writes that if a person says that an object is beautiful, then "... he does not count on the agreement of others with his judgment of satisfaction because he has frequently found them to be agreeable with his own, but rather demands it from them." (Kant 1790, §§18-22)
} 
To fit in these examples, the following provisos should be made. Firstly, the domain of agents to which the Universality Principle applies can vary. Usually, it is a community to which both the speaker and the addressee belong, but sometimes it is a community to which only one of them belongs, or neither. It need not even be a community of people. Secondly, the Experience Principle only applies to speaker and/or addressee if they belong to the domain of the Universality Principle.

In Example (17) the Universality Principle just comprises the students (plus maybe other people who are in the same stage of education). They are expected to find the exercise difficult. And since they are the addressees, they are subjected to the Experience Principle, and therefore should not accept the statement unless they do indeed find the exercise difficult.

In Example (18) neither the speaker (Sam), nor the addressee (Mary) are expected to find the new brand of cat food tasty, because they do not belong to the domain of the Universality Principle. Cat food is meant for cats, so cats form the domain of the Universality Principle. Sam is not sure the cat food is tasty, he just "thinks" so. But if he were sure, he would expect all cats to find it tasty. ${ }^{34}$

\section{The formal model, preliminaries}

\subsection{The framework of update semantics}

In this and the next few sections we will bring the ingredients presented informally above together in a formal model, which is built in the framework of update semantics, and accordingly takes the meaning $\llbracket \varphi \rrbracket$ of a sentence $\varphi$ to be an operation on intentional states.

Here is an overview of the key notions of the system.

\section{Definition 1 (Update system)}

- An update system for a language $\mathcal{L}$ is given by:

a. A set $\Sigma$ of intentional states.

b. A partial function $\llbracket . \rrbracket$ which, when defined, assigns a state $S \llbracket \varphi \rrbracket$ to a sentence $\varphi$ of $\mathcal{L}$ and a state $S \in \Sigma$.

- Sometimes the information conveyed by $\varphi$ will already be subsumed by $S$. In this case, we say that $\varphi$ is accepted in $S$, or that $S$ supports $\varphi$, and we write this as

\footnotetext{
34 The reader may think here of the exocentric perspective in truth-assessment discussed by Lasersohn (2005), cases where we assess "sentences for truth relative to contexts in which someone other than ourselves is specified as the judge, or regarding our assertions as justified by virtue of their truth relative to such contexts." (p. 670) He gives as examples free indirect discourse, "sentences in which a predicate such as fun is ascribed to a particular event" (p. 671) and questions. Pearson (2013) shows that the distinction of auto- vs exocentric cases is unclear. In the dynamic perspective we adopt, one can see that what is called exocentricity is not a distinctive feature of predicates of personal taste; when speaker and addressee are obviously non-peers, what matters is really the addressee's position. This way of handling this phenomenon puts it closer to similar instances of consistent but incoherent discourse.
} 
$S \models \varphi$. This relation can be defined as:

$$
S \models \varphi \text { iff } S \llbracket \varphi \rrbracket=S
$$

- An argument is valid iff whenever the update of a state with the premises is defined, the result is a state that supports the conclusion. Formally:

$\varphi_{1}, \ldots, \varphi_{n} \models \psi$ iff $S \llbracket \varphi_{1} \rrbracket \ldots \llbracket \varphi_{n} \rrbracket \models \psi$ for every $S$ such that $S \llbracket \varphi_{1} \rrbracket \ldots \llbracket \varphi_{n} \rrbracket$ is defined.

- A discourse $\varphi_{1}, \ldots, \varphi_{n}{ }^{35}$ is consistent iff there is some $S \in \Sigma$ such that $S \llbracket \varphi_{1} \rrbracket \ldots \llbracket \varphi_{n} \rrbracket$ is defined and $S \llbracket \varphi_{1} \rrbracket \ldots \llbracket \varphi_{n} \rrbracket \neq \emptyset$.

Here, the empty set $\emptyset$ serves as the absurd state. (It is a state that agents will try to avoid because if they are in this state they are at a loss, cognitively speaking.)

- The sentence $\varphi$ presupposes the sentence $\psi$ iff for all $S, S \llbracket \varphi \rrbracket$ is defined only if $S \models \psi$.

\subsection{Syntax}

The languages we will be concerned with in this section are not very complex.

\section{Definition 2 (Language)}

- A language $\mathcal{L}$ has the following non-logical symbols:

a. a number of individual constants among which $i$, you, $(s)$ he;

b. a number of nouns;

c. a number of adjectives. The adjectives are subdivided in two ways: uniform vs pluriform adjectives, and weakly evaluative vs strongly evaluative adjectives.

- Every adjective $G$ has an antonym $\breve{G}$ that is also an adjective. By definition $\breve{G}=G$.

- The set of sentences $\varphi$ of $\mathcal{L}$ is the smallest set including

a. Sentences of the form $N(c)$ for $N$ a noun, and $c$ an individual constant.

b. Unrestricted judgments $G(a), G-\operatorname{er}(a, b)$ for $G$ an RGA, and $a, b$ individual constants.

c. Restricted judgments Find $(G)(x, c)$, and Find $(G$-er $)(x, a, b)$, where $x \in$ $\{i$, you, $(s) h e\}$.

d. Sentences of the form $\neg \varphi$, for every sentence $\varphi$ defined above.

The languages $\mathcal{L}$ defined above do not have formulas representing sentences in which the adjective occurs in an attributive position. This is a shortcoming. As we saw in Sect. 2.3, the nouns $N$ in sentences of the form $x$ is a $G N$ play an important role in the process of interpretation as they often set the comparison basis and/or the comparison class. However, for the purposes of this paper it is not necessary to study these mechanisms in detail.

Note that Find does not take an agent and a sentence as its arguments-we did not write Find $\left(x,(G(c))\right.$. That is what we would have done if we thought ${ }^{36}$ that a sentence

\footnotetext{
35 By "A discourse $\varphi_{1}, \ldots, \varphi_{n}$ ", we just mean a sequence of sentences $\left\langle\varphi_{1}, \ldots, \varphi_{n}\right\rangle$.

36 As for instance Stephenson (2006, 2007) does.
} 
of the form $x$ finds $c G$ expresses a propositional attitude. However, on our account Find $(G)$ denotes a relation between agents and objects; Find $(G)(x, c)$ expresses an affective attitude of the agent $x$ with respect to the object $c$.

To see that $I$ find this tasty is not equivalent to I find that this is tasty, think of a newborn baby, Anna. One can easily say: Look! Anna finds infant formula tasty. But not: Look! Anna finds that infant formula is tasty. While the latter implies the former, the converse is not true.

\subsection{States}

The next definition introduces the main building blocks for the intentional states.

\section{Definition 3 (Situation)}

Let $\mathcal{L}$ be a language. Let $b(G)$ for every adjective $G$ be a nonempty set of nouns. Fix a set $D$ with a subset $A=$ \{speaker, addressee $\} \cup O$. D is called the domain, $A$ is called the set of agents. $O \neq \varnothing$, and is called the community.

A situation based on $D, A$ and $b$ is a pair $\langle I, T\rangle$, where:

- $I$ is a function that assigns

a. to each individual constant $c$ : an object $I(c) \in D ; I(i)=$ speaker, $I($ you $)=$ addressee, $I((s) h e) \in O$;

b. to each noun $N$ : a total function $I(N)$ from $D$ into $\{$ yes, no $\}$; if $N \in b(G)$ for some uniform $G$, and $I(N)(d)=$ yes, then for all $N^{\prime} \in b(G)$ with $N^{\prime} \neq N$, $I(N)(d)=$ no;

c. to each triple consisting of an adjective $G$, a noun $N \in b(G)$, and a set $C \subseteq\{d \in D \mid I(N)(d)=$ yes $\}$ : a partial function $I(G, N, C)$ from $C$ into $\{$ yes, no $\}$;

d. to each pair consisting of a comparative $G$-er and a noun $N \in b(G)$ : a partial function $I(G$-er,$N)$ from $\left\{\left\langle d, d^{\prime}\right\rangle \in D \times D \mid I(N)(d)=I(N)\left(d^{\prime}\right)=\right.$ yes $\}$ into $\{\mathbf{y e s}, \mathbf{n o}\}$.

$-T$ is a function that assigns

a. to each triple consisting of an adjective $G$, a noun $N \in b(G)$, and an agent $x \in A$ : a partial function $T(G, N, x)$ from $\{d \in D \mid I(N)(d)=$ yes $\}$ into $\{$ yes, no\};

b. to each triple consisting of a comparative $G$-er, a noun $N \in b(G)$ and an agent $x \in A$ : a partial function $T(G-e r, N, x)$ from $\left\{\left\langle d, d^{\prime}\right\rangle \in D \times D \mid I(N)(d)=\right.$ $I(N)\left(d^{\prime}\right)=$ yes $\}$ into $\{$ yes, no $\}$.

Some explanatory remarks are in order.

In natural language, RGA's like tasty are not only predicated of individual objects as given by, e.g., this cake, this piece of young Gouda, but also of the kind of entities given by mass nouns, e.g., chocolate cake, young Gouda, herring. For the sake of simplicity of the formalisation, the domain of objects in our models $D$ will only comprise individual objects.

The addressee is the owner of the state-it is her state that is being updated. The community $O$ consists of the agents who in the context at hand are subjected to 
the Universality Principle. Usually both the speaker and the addressee belong to this community.

Situations do not only record the interpretation given to the adjectives and the comparatives - modelled by the function I - but they also record tests - modelled by the function $T$ - which record the information about the affective responses of the agents.

The values $\{$ yes, no $\}$ constituting the range of the interpretation function $I$ signal the responses of the addressee. The value yes stands for agreement, and no for disagreement. We sometimes agree with someone, in particular with what she says, because we believe that what she says is true. We sometimes agree with someone because our responses are similar, for instance, when we listen to a piece music and we both love it. The values $\{\mathbf{y e s}, \mathbf{n o}\}$ are used to model our reactions in this wide sense of the words "agreement" and "disagreement".

For simplicity, nouns are given a total interpretation. This way, we restrict ourselves to cases where the addressee has complete information about the kinds of object constituting the domain. Of course, in reality this is seldom the case, and a full development of the system would have to explain how exactly one proceeds when this information is incomplete. But this way we can focus on what is our main concern: the interpretation of RGA's.

Let $G$ be some RGA. Suppose $N$ determines a comparison basis for $G$, and let $C \subseteq\{d \in D \mid I(N)(d)=$ yes $\}$ be a comparison class based on $N$. The interpretation function $I$ specifies which $d \in C$ the addressee has judged to be $G$-in which case $I(G, N, C)(d)=$ yes-and which $d \in C$ the addressee has judged to not be $G$-in which case $I(G, N, C)(d)=$ no. There may be a lot of objects $d \in C$ that have not yet been evaluated. Then $I(G, N, C)(d)$ is undefined.

Similar remarks apply to the interpretation of the comparative $G$-er. But as we noted in Sects. 2.1 and 2.2, for the interpretation of comparatives only the comparison basis matters.

The $T$ function records for each $G$ and comparison basis $N$ which objects the addressee finds $G$. The " $T$ " stands for testing, which in the case of the adjective tasty amounts to tasting.

Different RGA's come with different tests, and for a particular adjective $G$ the test will vary with the comparison basis. The comparison classes do not come in because the outcome of the kind of test we are discussing here is not based on a comparison. Test functions are concerned with the relation between subject and object: it matters what kind of object this is, but that is all. Previous experiences have surely shaped and developed the subject's taste in the course of time, but at a given moment, when the subject is asked whether she finds a particular object $G$, it is between her and the object, it is about how this object affects her. As we already mentioned in our discussion of the Müller-Lyer case in Sect. 3.4, one cannot be mistaken about how things appear to oneself.

The incorporation of these tests in the states enables us to integrate affective responses into the semantics. A statement of the form I find $x G$ expresses an affective response. It does not, as many working in a degree-based framework are inclined to 
think, report that the degree of tastiness of $x$ on one's private tastiness scale is higher than one's private standard of tastiness. ${ }^{37}$

Anybody acquainted with Wittgenstein's private language argument should frown at the notions of a 'private scale' and 'private standard'. But even if one thinks that these are coherent notions, one should be aware that, starting from such an analysis, it is difficult to understand why an agent cannot be mistaken about what she finds. After all, it could be that an agent thinks that the degree of tastiness on her private tastiness scale is higher than her private standard of tastiness, whereas in fact it isn't. Or are there no facts in this private matter?

We are now ready to define the notion of an intentional state - or at least the aspects of such states that matter to the interpretation of RGA's.

\section{Definition 4 (Basic state)}

A basic state $\sigma$ based on $D, A$ and $b$ has two components, $\sigma=\left\langle\alpha_{\sigma}, \epsilon_{\sigma}\right\rangle$. Both $\alpha_{\sigma}$ and $\epsilon_{\sigma}$ are situations based on $D, A$ and $b$. They are related as follows.

a. For every noun $N, I_{\alpha_{\sigma}}(N)=I_{\epsilon_{\sigma}}(N)$.

b. For every adjective $G, I_{\alpha_{\sigma}}(G, N, C) \subseteq I_{\epsilon_{\sigma}}(G, N, C)$.

The situation $\alpha_{\sigma}$ models the current evaluations, $\epsilon_{\sigma}$ models the expectations about further evaluations. When $\alpha_{\sigma}$ and $\epsilon_{\sigma}$ increase, the state $\sigma$ gets stronger in the following sense.

Definition 5 Let $\sigma=\left\langle\alpha_{\sigma}, \epsilon_{\sigma}\right\rangle$ and $\sigma^{\prime}=\left\langle\alpha_{\sigma}^{\prime}, \epsilon_{\sigma}^{\prime}\right\rangle$ be basic states based on the same $D$, $A$ and $b$. The state $\sigma^{\prime}$ is at least as strong as $\sigma$ iff $I_{\alpha_{\sigma}} \subseteq I_{\alpha_{\sigma^{\prime}}}, I_{\epsilon_{\sigma}} \subseteq I_{\epsilon_{\sigma^{\prime}}}, T_{\alpha_{\sigma}} \subseteq T_{\alpha_{\sigma^{\prime}}}$, and $T_{\epsilon_{\sigma}} \subseteq T_{\epsilon_{\sigma^{\prime}}}$.

\section{Some constraints}

\subsection{General constraints}

Here are some general constraints on the interpretation functions $I_{\alpha_{\sigma}}$ and $I_{\epsilon_{\sigma}}$ figuring in a state $\left\langle\alpha_{\sigma}, \epsilon_{\sigma}\right\rangle$. An agent should not accept a statement if doing so would turn her state into a state in which one or more of these constraints are violated. In other words, ideally, every state will satisfy these constraints.

\section{Definition 6 (General constraints)}

- Transitivity: If $I(G-e r, N)\left(d, d^{\prime}\right)=$ yes and $I(G-e r, N)\left(d^{\prime}, d^{\prime \prime}\right)=$ yes, then $I(G-e r, N)\left(d, d^{\prime \prime}\right)=$ yes.

- Asymmetry: If $I(G$-er,$N)\left(d, d^{\prime}\right)=$ yes, then $I(G$-er,$N)\left(d^{\prime}, d\right)=$ no.

- Monotonicity: If $I(G, N, C)(d)=$ yes, and $I(G-e r, N)\left(d^{\prime}, d\right)=$ yes, then $I(G, N, C)\left(d^{\prime}\right)=$ yes.

- Comparativity:

a. If $I(G, N, C)(d)=$ yes and $I(G, N, C)\left(d^{\prime}\right)=$ no, then $I(G$-er, $N)\left(d, d^{\prime}\right)=$ yes;

37 Cf. e.g., Kennedy (2013), Barker (2013) and Sassoon (2010). 
b. If $I(\breve{G}, N, C)(d)=$ no and $I(\breve{G}, N, C)\left(d^{\prime}\right)=$ yes, then $I(G$-er, $N)\left(d, d^{\prime}\right)=$ yes.

- Antonymy:

a. If $I(G-e r, N)\left(d, d^{\prime}\right)=$ yes, then $I(\breve{G}-e r, N)\left(d, d^{\prime}\right)=$ no;

b. If $I(G, N, C)(d)=$ yes, then $I(\breve{G}, N, C)(d)=$ no.

- EOI: If $I(G$-er,$N)\left(d, d^{\prime}\right)=$ no and $I(G$-er,$N)\left(d^{\prime}, d\right)=$ no, then $I(G, N, C)(d)=$ yes $/$ no iff $I(G, N, C)\left(d^{\prime}\right)=$ yes $/$ no.

Ideally, for a given RGA $G$, comparison basis $N$, and comparison class $C$, the end result of the evaluation process would be a partition of $C$ in a number of equivalence classes, each consisting of objects that are equally $G$. These equivalence classes should be linearly ordered from the equivalence class containing the $G$-est objects on the one end, to the equivalence class containing the $\breve{G}$-est objects on the other end. There can be several equivalence classes containing objects that are $G$ (some containing objects that are very $G$, other containing objects that are extremely $G$, etc.), several equivalence classes containing objects that are $\breve{G}$, and in-between these there may be a number of equivalence classes containing objects that are neither $G$ nor $\breve{G}$.

As we already saw in Sect. 3.3, this ideal is not always attainable. In practice, and in line with EOI, our agents will consider two objects with property $N$ equally $G$ iff there is no observational difference between them: both $I(G-e r, N)\left(d, d^{\prime}\right)=$ no and $I(G-e r, N)\left(d^{\prime}, d\right)=$ no. There is nothing wrong with this, as long as the following holds:

(*) For all $d, d^{\prime}, d^{\prime \prime} \in C$, if $I(G-e r, N)\left(d, d^{\prime}\right)=$ no and $I(G-e r, N)\left(d^{\prime}, d^{\prime \prime}\right)=$ no, then $I(G-e r, N)\left(d, d^{\prime \prime}\right)=$ no.

Proposition 7 Let $\approx_{G, N}$ for every $G$ and $N \in b(G)$ be a relational predicate, and define:

$$
I\left(\approx_{G, N}\right)\left(d, d^{\prime}\right)=\text { yes iff } I(G-e r, N)\left(d, d^{\prime}\right)=\text { no and } I(G-e r, N)\left(d^{\prime}, d\right)=\text { no. }
$$

Suppose $I(G$-er, $N)$ is total. Then, given Transitivity and Asymmetry, $I\left(\approx_{G, N}\right)$ is an equivalence relation on $N$ iff $(*)$ holds.

The problem is that in many comparison classes, $(*)$ will not hold. This means that in many cases one cannot clearly distinguish equivalence classes. There are no sharp borderlines, things are blurred. And as we already saw, in the worst case this may lead to the Sorites paradox. But again, there is no reason to panic here. The fact that in some cases the machinery runs down does not mean that the machinery is useless.

In many cases the machinery works well because in many cases the agents evaluate only a few objects. As long as they do not have to rate each and every object as $G$ or not $G$, and fully specify which objects are $G$-er than which other objects, things will go well. They only get into trouble if they are confronted with the kind of chain we described in Sect. 3.3, and have to rate all objects on the chain as $G$ or not $G$. Then the classificatory tools supplied by RGA's fail. RGA's can only give rough classifications, but such chains do not lend themselves to rough classification. 
(Note that - metaphorically speaking - in a truth-conditional set-up, the truth definition has to 'rate' every object as $G$ or not $G$, and must specify completely which objects are $G$-er than which other objects, and therefore cannot live up to the constraints mentioned above if (*) does not hold. That is how the paradox arises.)

The constraints for the positive form of an adjective $G$ all concern the interpretation of $G$ in a specific context $\langle N, C\rangle$. So, it might seem that the interpretations $I(G, N, C)$ and $I\left(G, N, C^{\prime}\right)$ of $G$ in two different comparison classes $C$ and $C^{\prime}$ are independent from one another. However, the interpretation of the comparative $G$-er does not depend on the comparison class but is defined on the comparison basis. This has cross-contextual effects. For example, Comparativity together with Asymmetry imply the following:

Proposition 8 (No reversal)

If $I(G, N, C)(d)=$ yes and $I(G, N, C)\left(d^{\prime}\right)=$ no, then for no $C^{\prime} \subseteq I(N)$, $I\left(G, N, C^{\prime}\right)\left(d^{\prime}\right)=$ yes and $I\left(G, N, C^{\prime}\right)(d)=$ no.

Finally, all the constraints defined above are imposed on the interpretation function $I$. What holds for $I$, need not hold for $T$. The Transitivity constraint says that an agent who accepts that $x$ is tastier than $y$, and that $y$ is tastier than $z$, has to accept that $x$ is tastier than $z$. But an agent who finds $x$ tastier than $y$, and who finds $y$ tastier than $z$, does not have to find $x$ tastier than $z$. Not by any logical principle, at least.

\subsection{Special constraints}

The constraints given above hold for all RGA's. The next one is specific for strongly evaluative adjectives. It is a formalisation of the Universality Principle discussed informally in Sect. 3.5.

\section{Definition 9 (Universality principle)}

Let $\mathcal{L}$ be a language and $\sigma=\left\langle\alpha_{\sigma}, \epsilon_{\sigma}\right\rangle$ a basic state. Let $G$ be a strongly evaluative adjective of $\mathcal{L}$.

a. If $I_{\alpha_{\sigma}}(G, N, N)(d)$ is defined, then for all $x \in O$, $T_{\epsilon_{\sigma}}(G, N, x)(d)=I_{\alpha_{\sigma}}(G, N, N)(d)$.

b. If $I_{\alpha_{\sigma}}(G-e r, N)\left(d, d^{\prime}\right)$ is defined, then for all $x \in O$, $T_{\epsilon_{\sigma}}(G-e r, N, x)\left(d, d^{\prime}\right)=I_{\alpha_{\sigma}}(G-e r, N)\left(d, d^{\prime}\right)$.

If according to you a certain painting is beautiful, you will expect everybody in the community $O$-which usually is just 'your' community-to find it beautiful. That is what the above principle enforces. As we indicated in Sect. 3.5 the expectations involved are not just epistemic. They also have a normative side. You will not just presume that people who have not yet seen the painting will find it beautiful. You will also expect people that did not find the painting beautiful to change their mind. So, if $I_{\alpha_{\sigma}}(G, N, N)(d)=$ yes, then also if $T_{\alpha_{\sigma}}(G, N, x)(d)=$ no, $T_{\epsilon_{\sigma}}(G, N, x)(d)=$ yes.

The Universality Principle only pertains to $I_{\alpha_{\sigma}}(G, N, N)(d)$, and not to $I_{\alpha_{\sigma}}(G, N, C)(d)$ for $C \neq N$. When $I(G, N, N)(d)=$ yes, this means that $d$ is judged $G$ relative to all objects of the comparison basis $N$. This fits in with the idea that the test function $T_{\alpha_{\sigma}}$ only depends on $N$. 
The next definition formalises the other of the aspects of Kant's analysis of taste judgments, which we discussed informally in Sect. 3.4.

\section{Definition 10 (Experience principle)}

If $G$ is strongly evaluative, the following holds:

a. If $d \in \operatorname{dom} I_{\alpha_{\sigma}}(G, N, N),{ }^{38}$ and addressee $\in O$, then $d \in \operatorname{dom} T_{\alpha_{\sigma}}(G, N$, addressee $)$, and $I_{\alpha_{\sigma}}(G, N, N)(d)=T_{\alpha_{\sigma}}(G, N$, addressee $)$ $(d)$.

b. If $\left\langle d, d^{\prime}\right\rangle \in \operatorname{dom} I_{\alpha_{\sigma}}(G-e r, N)$, and addressee $\in O$, then $\left\langle d, d^{\prime}\right\rangle \in \operatorname{dom} T_{\alpha_{\sigma}}(G$-er, $N$, addressee $)$ and $I_{\alpha_{\sigma}}(G$-er, $N)\left(d, d^{\prime}\right)=$ $T_{\alpha_{\sigma}}(G$-er, $N$, addressee $)\left(d, d^{\prime}\right)$.

The Experience Principle does not hold in the exceptional case that the addressee does not belong to the community that is subjected to the Universality Principle. But if she does, she cannot agree with the statement that $x$ is $G$ unless she has tested $x$ for herself, and found it $G$.

The Müller-Lyer example illustrates that the Experience Principle only holds for strong evaluatives. Still, if your experience does not correspond to what you believe to be actually the case, you will not feel at ease, psychologically speaking. To be more precise, in a harmonious state there are no $G, N$, and $d \in D$ such that $I_{\alpha_{\sigma}}(G, N, N)(d)=$ yes $/$ no while $T_{\alpha_{\sigma}}(G, N$, addressee $)(d)=\mathbf{n o} / \mathbf{y e s}$ (and similarly for the comparative $G$-er).

Disharmonious states are relatively rare. To a large extent, our experience and knowledge about the world remain in tune with one another.

The last constraint we will impose on our states pertains to weak evaluatives. To formulate it, we need the notion of minimal state.

\section{Definition 11 (Minimal state)}

Fix a domain $D$, and an interpretation $I$ for the nouns $N$. The minimal state $\mu$ based on $A, D$, and $I$ is a basic state $\left\langle\alpha_{\mu}, \epsilon_{\mu}\right\rangle$, in which the following holds:

a. for all nouns $I_{\alpha_{\mu}}=I_{\epsilon_{\mu}}=I(N)$;

b. for all adjectives $G$, nouns $N \in b(G)$, and $x \in A$, $\operatorname{dom} I_{\alpha_{\mu}}(G$-er, $N)=$ $\operatorname{domI}_{\epsilon_{\mu}}(G$-er,$N)=\operatorname{dom}_{\alpha_{\mu}}(G$-er, $N, x)=\operatorname{dom}_{\alpha_{\mu}}(G-e r, N, x)=\emptyset$;

c. for all adjectives $G$, nouns $N \in b(G), C \subseteq\{d \in D \mid I(N)(d)=$ yes $\}$, and $x \in A$, $\operatorname{domI}_{\alpha_{\mu}}(G, N, C)=\operatorname{dom}_{\alpha_{\mu}}(G, N, C, x)=\emptyset$

d. Normality: for all weakly evaluative $G$ and $d \in D, I_{\epsilon_{\mu}}(G, N, C)(d)=$ $T_{\epsilon_{\mu}}(G, N, C, x)(d)=$ no and $I_{\epsilon_{\mu}}(\breve{G}, N, C)(d)=T_{\epsilon_{\mu}}(\breve{G}, N, C, x)(d)=\mathbf{n o}$;

e. for all strongly evaluative $G, \operatorname{domI}_{\epsilon_{\mu}}(G, N, C)=\operatorname{dom}_{\epsilon_{\mu}}(G, N, C, x)=\emptyset$.

The definition provides a useful abstraction, as the minimal state shows how we would proceed if we had to start the evaluation process from scratch. For weak evaluatives, we start out expecting things to be neither $G$ nor $\breve{G}$. Hence, a sentence of the form $x$ is $G$ will express something one would not expect, something surprising,

$\overline{38 \operatorname{dom} I_{\alpha_{\sigma}}(G, N, C) \text { is the set of all } d \in C \text { for which } I_{\alpha_{\sigma}}(G, N, C)}(d)$ is defined. 
and therefore worth mentioning. In the rules defined below, we build in that we keep expecting things to be neither $G$ nor $\breve{G}$ until we learn otherwise. ${ }^{39}$

\subsection{For further discussion}

With the above, the list of special constraints is not exhausted. Here is one example of a constraint one might want to add:

- Conversion: If $G$ is weakly evaluative, and $I(G$-er, $N)\left(d, d^{\prime}\right)=$ yes, then $I(\breve{G}$-er,$N)\left(d^{\prime}, d\right)=$ yes. $^{40}$

If you accept that $x$ is taller than $y$ you will also also have to accept that $y$ is shorter than $x$, and it looks like one can substitute any weak evaluative and its antonym for tall and short here. But as beautiful and its antonym ugly illustrate Conversion does not hold for strong evaluatives. If you accept that Venice is more beautiful than Amsterdam, you don't have to accept that Amsterdam is uglier than Venice. The latter implies that Amsterdam is ugly, the former does not. Actually, this last observation is in line with another special constraint:

- Projectivity: If $G$ is strongly evaluative, and $I(G-e r, N)\left(d, d^{\prime}\right)=$ yes, then $I(G, N, N)(d)=$ yes.

We will not try to complete the list. To do so in a systematic way would lead us too far away from the central issues in this paper.

Closer to the central issues is the following. Compare:
a. In the seventies the university built some ugly dormitories, ...
b. \# ...but I don't find them ugly.
c. ...but I did not find them ugly at the time.
a. The apple pie we had yesterday was tasty, ...
b. \# ... but I didn't find it tasty.

It looks like the Experience Principle works differently in (19) than in (20). Example (19) suggests that if you talk about the past, and say about things in the past that they were ugly, then the point in time at which this evaluation takes place is the utterance time. That is why (19-b) sounds strange. Given (19-a), the speaker should find the dormitories ugly now-even if they have been teared down in the meantime. For tasty, the evaluation takes place at reference time. At least that is what (20) suggests. You cannot sincerely say that the pie you had was tasty if you did not find it tasty at the time you tasted it.

We have not looked into this matter any further. It would be interesting to extend these observations to other strong evaluatives, and even more interesting to bring in time in our model and to see how these examples and the closely connected Examples (13) and (14) can be formally dealt with.

\footnotetext{
39 In Sect. 3.2, we discussed normality for weak evaluatives, so we formulate the Normality constraint only for weak evaluatives. We remain agnostic with regard to its application to strong evaluatives. This is what the (e) clause takes care of.

40 Given Asymmetry, this constraint is a strengthening of Antonymy.
} 


\section{Updates}

\subsection{Updating with unrestricted judgments}

We are ready now to discuss the update conditions of unrestricted judgements, i.e., sentences of the form $x$ is $G$.

Consider first a sentence of the form $G(a)$ with $G$ weakly evaluative. For this sentence to be interpretable, it has to be clear to the addressee what the relevant comparison basis $N$ and comparison class $C$ are. Then updating the state $\sigma$ in the context $\langle N, C\rangle$ starts with setting the value of $I_{\alpha_{\sigma}}(G, N, C)(I(a))$ to yes. Of course, if the addressee cannot do so without getting in conflict with the constraints set on the use of $G$, the statement should not be accepted. Suppose, for example, that $I_{\alpha_{\sigma}}(G, N, C)(I(b))=$ no and $I_{\alpha_{\sigma}}(G$-er, $N)(b, a)=$ yes. Then setting $I_{\alpha_{\sigma}}(G, N, C)(I(a))$ to yes conflicts with Monotonicity.

Now, suppose that no such conflicts arise. Then in many cases accepting $G(a)$ will lead to more changes. The new state will have to satisfy the constraints. Again, think of Monotonicity. If you extend $I_{\alpha_{\sigma}}(G, N, C)$ with the pair $\langle I(a)$, yes $\rangle$, and $I_{\alpha_{\sigma}}(G$-er,$N)(c, a)=$ yes, then you will have to add the pair $\langle I(c)$, yes $\rangle$ to $I_{\alpha_{\sigma}}(G, N, C)$ as well (assuming it is not already there).

Spelling this out formally is rather cumbersome. Before we do so, we note the following.

Proposition 12 Let $\mathcal{I}$ be some set of interpretations such that each $I \in \mathcal{I}$ satisfies the constraints. Consider I' defined by:

- $I^{\prime}(G, N, C)(d)=$ yes iff for every $I \in \mathcal{I}, I(G, N, C)(d)=$ yes;

- $I^{\prime}(G, N, C)(d)=$ no iff for every $I \in \mathcal{I}, I(G, N, C)(d)=$ no;

Then I' satisfies the constraints.

Proof The proof is straightforward.

The proposition implies that if there exist extensions of $I(G, N, C)$ with the pair $\langle I(a)$, yes $\rangle$ that satisfy the constraints, then there is a smallest extension with these properties. Hence, if the interpretation function can be adapted to the constraints, we can rest assured there is a unique minimal way to do so. Given this, the following is a proper definition.

Definition 13 (Update rules for weak evaluatives)

Let $\sigma=\langle\alpha, \epsilon\rangle$ be a basic state. $\sigma \llbracket G(a), N, C \rrbracket$, the result of updating the state $\sigma$ in the context given by the comparison basis $N$ and the comparison class $C$, is determined as follows:

- $\sigma \llbracket G(a), N, C \rrbracket=\emptyset$ if there is no extension $J$ of $I_{\alpha_{\sigma}}$ such that $J(G, N, C)(I(a))=$ yes and all constraints are satisfied.

- Otherwise, $\sigma \llbracket G(a), N, C \rrbracket=\sigma^{\prime}$, where $\sigma^{\prime}$ is determined as follows:

a. $I_{\alpha_{\sigma^{\prime}}}$ is the smallest extension $J$ of $I_{\alpha_{\sigma}}$ such that $J(G, N, C)(I(a))=$ yes and all constraints are satisfied.

b. $T_{\alpha_{\sigma^{\prime}}}=T_{\alpha_{\sigma}}$. 
c. $I_{\epsilon_{\sigma^{\prime}}}$ is the smallest extension $J$ of $I_{\alpha_{\sigma^{\prime}}}$ such that:

i. $J\left(G, N, C^{\prime}\right)(d)=$ yes/no for all $d$ and $C^{\prime}$ such that $I_{\alpha_{\sigma^{\prime}}}\left(G, N, C^{\prime}\right)(d)$ is undefined and $I_{\epsilon_{\sigma}}\left(G, N, C^{\prime}\right)(d)=$ yes $/ \mathbf{n o}$;

ii. $J(G$-er, $N)\left(d, d^{\prime}\right)=\mathbf{y e s} / \mathbf{n o}$ for all $d, d^{\prime}$ such that $I_{\alpha_{\sigma^{\prime}}}(G$-er,$N)\left(d, d^{\prime}\right)$ is undefined and $I_{\epsilon_{\sigma}}(G$-er,$N)\left(d, d^{\prime}\right)=$ yes $/$ no;

iii. the constraints are satisfied.

d. $T_{\epsilon_{\sigma^{\prime}}}=T_{\epsilon_{\sigma}}$.

We did not prepare the reader for the (c) clause in the definition above. It is there to make sure that the agents keep ${ }^{41}$ expecting things to be normal, if what they learn does not force them to think otherwise. When you have come to accept that Alf is tall, you will have to give up the expectation that he was neither tall nor short, but you may perhaps still go on expecting Bert is neither tall nor short. But notice that, by Comparativity, this means you will now expect Alf to be taller than Bert. And so, if by any chance the next thing you learn is that Bert is taller than Alf, you will have to give up this new expectation right away. The (c) clause takes care of this and similar situations.

The definition of $\sigma \llbracket G-\operatorname{er}(a, b), N, C) \rrbracket$ for the comparative $G$-er of a weakly evaluative $G$ follows the same pattern as the definition for $G$ given above, so we will not spell it out.

In the update condition for unrestricted judgments featuring strongly evaluative adjectives, we have to distinguish two cases depending on whether or not the addressee belongs to the community constituting the domain for the Universality Principle (cf. Definition 9). Only if the addressee belongs to this community, the Experience Principle applies (cf. Definition 10).

If addressee $\in O$, she can only accept or reject $G(a)$ if she has tested for herself whether she finds $a G$. Moreover, for the case that $C=\{d \in D \mid I(N)(d)=$ yes $\}$, it will be a necessary condition for $G(a)$ to be acceptable that this test had a positive outcome. This way the Experience Principle will be satisfied.

To satisfy the Universality Principle, the expectations in $T_{\epsilon_{\sigma}}$ have to be updated, so that the addressee will expect other members of the community to have a response to the test matching hers.

In other respects, the conditions are the same as for weak evaluatives.

Definition 14 (Update rules for strong evaluatives)

Let $\sigma=\langle\alpha, \epsilon\rangle$ be a basic state, and suppose addressee $\in O . \sigma \llbracket G(a), N, C \rrbracket$ exists only if $I(a) \in \operatorname{dom} T(G, N$, addressee $)$. If so, the following holds:

$-\sigma \llbracket G(a), N, C \rrbracket=\emptyset$ in each of the following cases:

a. $C=I(N)$ and $T_{\alpha_{\sigma}}(G, N$, addressee $)(I(a))=$ no, or

b. there is no extension $J$ of $I_{\alpha_{\sigma}}$ such that $J(G, N, C)(I(a))=$ yes and the basic constraints are satisfied, or

c. $C=I(N)$ and there is no extension $T$ of $T_{\epsilon_{\sigma}}$ such that for all $x \in O, T(G, N, x)(I(a))=$ yes.

\footnotetext{
41 Here, we assume that agents start out from the minimal state (cf. Definition 11), and that the only changes are changes resulting from the updates we are defining here.
} 
- Otherwise, $\sigma \llbracket G(a), N, C \rrbracket=\sigma^{\prime}$, where $\sigma^{\prime}$ is determined as follows:

a. $I_{\alpha_{\sigma^{\prime}}}$ is the smallest extension $J$ of $I_{\alpha_{\sigma}}$ such that $J(G, N, C)(I(a))=$ yes and the constraints are satisfied.

b. $T_{\alpha_{\sigma^{\prime}}}=T_{\alpha_{\sigma}}$.

c. $I_{\epsilon_{\sigma^{\prime}}}$ is the smallest extension $J$ of $I_{\alpha_{\sigma^{\prime}}}$ such that:

i. $J\left(G, N, C^{\prime}\right)(d)=$ yes/no for all $d$ and $C^{\prime}$ such that $I_{\alpha_{\sigma^{\prime}}}\left(G, N, C^{\prime}\right)(d)$ is undefined and $I_{\epsilon_{\sigma}}\left(G, N, C^{\prime}\right)(d)=$ yes $/$ no;

ii. $J(G$-er, $N)\left(d, d^{\prime}\right)=\mathbf{y e s} / \mathbf{n o}$ for all $d, d^{\prime}$ such that

$I_{\alpha_{\sigma^{\prime}}}(G$-er,$N)\left(d, d^{\prime}\right)$ is undefined and $I_{\epsilon_{\sigma}}(G$-er,$N)\left(d, d^{\prime}\right)=\mathbf{y e s} / \mathbf{n o}$;

iii. the constraints are satisfied.

d. If $C=I(N)$, then $T_{\epsilon_{\sigma^{\prime}}}$ is the smallest extension $T$ of $T_{\epsilon_{\sigma}}$ such that for all $x \in O, T(G, N, x)(I(a))=$ yes. Otherwise, $T_{\epsilon_{\sigma^{\prime}}}=T_{\epsilon_{\sigma}}$.

If addressee $\notin O$, the Experience Principle plays no role. It is left to the reader to adapt the definition to that case.

\subsection{Updating with restricted judgments}

The next definition deals with restricted judgments, i.e., sentences of the form $x$ finds y G, like I find this cake tasty, You find this suitcase heavy, or She finds this exercise difficult. The rules are the same for weak and strong evaluatives.

Definition 15 (Update rule for restricted judgments)

Let $\sigma=\langle\alpha, \epsilon\rangle$ be a basic state. $\sigma \llbracket \operatorname{Find}(G)(x, a), N \rrbracket$ is determined as follows:

a. Suppose $x=$ you.

- If $\langle I(a)\rangle \notin \operatorname{dom}(T(G, N$, addressee $)), \sigma \llbracket \operatorname{Find}(G)(y o u, a), N \rrbracket$ is not defined.

- If $T(G, N$, addressee $)(I(a))=$ yes, $\sigma \llbracket$ Find $(G)($ you, $a), N, C \rrbracket=\sigma$.

- And if $T(G, N$, addressee $)(I(a))=$ no, $\sigma \llbracket \operatorname{Find}(G)($ you, $a), N \rrbracket=\emptyset$.

b. Suppose $x \in\{i,(s) h e\}$.

- If $T(G, N, I(x))(I(a))=$ no, $\sigma \llbracket F i n d(G)(x, a), N \rrbracket=\emptyset$.

- Otherwise, $\sigma \llbracket$ Find $(G)(x, a), N \rrbracket=\sigma^{\prime}$ where $\sigma^{\prime}$ is determined as follows:

i. $I_{\alpha_{\sigma^{\prime}}}=I_{\alpha_{\sigma}}$

ii. $T_{\alpha_{\sigma^{\prime}}}$ is the smallest extension $T$ of $T_{\alpha_{\sigma}}$ such that

$T(G, N, I(x))(I(a))=\mathbf{y e s}$

iii. $I_{\epsilon_{\sigma^{\prime}}}=I_{\epsilon_{\sigma}}$;

iv. $T_{\epsilon_{\sigma^{\prime}}}=T_{\epsilon_{\sigma}}$.

Recall once again the addressee is the owner of the state, the referent of "you" in a statement of the form Find $(G)(y o u, a)$. Of course, it is hard to think of a context in which somebody different from yourself tells you what you find. In such a case, you can only accept the statement if you already accepted it: if you already took the test and the outcome was positive.

If the statement has the form $\operatorname{Find}(G)(i, a)$ or $\operatorname{Find}(G)((s) h e, a)$, the addressee may learn something new about the speaker or the other agents figuring in our model. 
Of course, if you have reasons to think that the speaker's or the other agent's behaviour is not in line with the statement, you should protest and reject the update, but in other cases, why not accept it?

\subsection{Adding negation}

To define update conditions for sentences of the form $\neg \varphi$, we have to complicate matters a bit. We must ensure that, after learning $\neg \varphi$, an update with $\varphi$ is impossible. In an eliminative set-up in which a state of partial information is given by the set of its complete extensions, this is easy to do. To update $\sigma$ with $\neg \varphi$, one can just subtract the set of complete extensions left open after an update with $\varphi$ from $\sigma$. But in the constructive set-up of this paper such a trick is not available.

Now, think of the updates that we have been dealing with so far as positive updates, updates that result in accepting a certain statement. Let's write $\sigma \llbracket \varphi \rrbracket^{+}$rather than $\sigma \llbracket \varphi \rrbracket$ for these. Parallel to these, we will define negative updates, updates that result in rejecting a statement, and write $\sigma \llbracket \varphi \rrbracket^{-}$for these. Once they are available, we can define a positive update with $\neg \varphi$ as a negative update with $\varphi$ and vice versa.

$$
\begin{aligned}
& \sigma \llbracket \neg \varphi \rrbracket^{+}=\sigma \llbracket \varphi \rrbracket^{-} \\
& \sigma \llbracket \neg \varphi \rrbracket^{-}=\sigma \llbracket \varphi \rrbracket^{+}
\end{aligned}
$$

It is pretty straightforward to specify the negative correlates of Definitions 13, 14, and 15. Just replace the occurrences of yes in these definitions by an occurrence of no, and vice versa.

In most truth-conditional systems dealing with vague adjectives, a sentence like John is tall can be true, false, or undefined, where the latter stands for neither (definitely) tall, nor (definitely) not tall, but something in-between, a borderline case. That is not how things work out here. Here, when John is 'something in-between', this does not mean that John is something in-between tall and not tall, but something in-between tall and short. In this case, the agents reject both John is tall and John is short, and accept both John is not tall and John is not short. When in the system presented here an agent accepts neither John is tall, nor John is not tall, this is because the agent has no information about the question whether or not John is tall. She accepts neither, not because she thinks John is a borderline case, but because she has no idea what John's height is. ${ }^{42}$

At several places in this paper, we have given examples suggesting that if $G$ is strongly evaluative, there is no state supporting both $x$ is $G$ and I don't find $x G$.

Now, strictly speaking this only holds in the following circumstances:

a. The speaker belongs to the community that forms the domain $O$ of the Universality Principle.

b. The speaker is sincere, i.e., she only asserts sentences that are supported by her state.

\footnotetext{
42 A possible source of confusion is the fact both approaches give rise to a partial logic, in which the law of excluded middle does not hold. The truth-conditional systems give rise to a partial logic because there are truth value gaps. The update system does so because there may be gaps in an agent's information.
} 
And what we can prove is, more precisely, that if (a.) and (b.) hold, the speaker's state cannot support both This is $G$ and I don't find this G. So, on our account This is tasty but I do not find it tasty is a pragmatic contradiction. We need the maxim of quality to explain why it is out.

Another subtlety to keep in mind is that when we investigate the speaker's state to see if it supports a given sentence, we are dealing with a reflective state, a state in which the speaker and the addressee are the same person.

Here is the proof: given Definition 14, and more specifically the formalisation of the Experience Principle, if addressee $\in O$, then her state $\sigma$ can only support $G(\mathrm{a})$ if $T_{\alpha_{\sigma}}(G$, addressee $)(I(a))=$ yes, whereas according to the negative analogue of Definition 15, $\neg \operatorname{Find}(G)(i, I(a))$ is only supported by a state $\sigma$ if $T_{\alpha_{\sigma}}(G$, speaker $)(I(a))=$ no. In a reflective state this yields a contradiction.

It is instructive to compare $x$ is $G$ but I do not find $x G$, with $x$ is $G$ but you don't find $x$ $G$. The latter can be sincerely asserted (probably the speaker would put it the other way around: (I heard) You don't find this tasty, but it is), but the addressee cannot agree. If the speaker is right about the addressee's assessment, then the addressee cannot agree with $x$ is $G$, and if the speaker is wrong about the addressee's assessment then of course the addressee will also have to say No! (Maybe it comes as a surprise that there exist sentences that a speaker can accept and sincerely assert but an addressee cannot accept. However, this is not the only example; think of sentences like: It is raining even though you don't believe it.)

Most researchers that have been working with update semantics think of the update rules as rules for updating the common ground. We don't. We think of the update rules as rules for the addressee. Updating is about processing and interpreting new information. The natural agent for doing so is the addressee. In a recent paper (Crespo et al. 2018) we argue that is the right way to look at it, in particular if you want to get to grips with the notion of presupposition.

The reader may have noticed that the Experience Principle works out as a presupposition in the sense of Definition 4. Updating with $x$ is $G$ presupposes that the addressee (if s/he belongs to the community $O$ ) has taken the relevant test. If not, there is no update; the addressee can neither accept nor reject the statement.

Nowadays presuppositions are mostly understood as speaker's presuppositions, necessary conditions for a sentence to be accepted or rejected by the speaker. Ninan (2014) discusses the question whether what he calls the 'acquaintance inference' can be considered a presupposition in this sense of the word. He is hesitant to do so because this inference does not have the projection properties that ordinary presuppositions have. It projects under negation, but it does not project under questions. The question:

Are the lobster rolls tasty?

does not presuppose that the speaker has tasted the lobster. But then, as Ninan notes: "it does suggest that the hearer has." (Ninan 2014, p. 299) And that is exactly our point. Roughly speaking, given the standard theory about the semantics of questions (Groenendijk and Stokhof 1984), updating with a yes/no question amounts to splitting the state in two parts, one part the result of an update with the positive answer, and the other the result of an update with the negative answer. Both answers presuppose 
that the test has been taken. So, this means that the addressee can only accept the question as a decent question if she has tried the lobster rolls, because only then can she answer it. In other words, if the notion of presupposition is defined the way we defined it, there is no problem showing that this particular presupposition is projected under questions.

This is not the last word about this topic. There are more issues to discuss. For example, It should be possible for the addressee to accept $x$ might be tasty in a context in which she has not yet tasted $x$. So, the presupposition imposed by the Experience Principle does not project under epistemic might. ${ }^{43}$ Given this, maybe some readers wouldn't want to call it a presupposition after all.

\subsection{Conjunction, disjunction, and other logical constants}

Extending the system with conjunction, disjunction and other logical constants brings up a host of new issues. This section is meant to briefly sketch a number of them.

A disjunction $\varphi \vee \psi$ introduces two (not necessarily exclusive) alternatives. In this constructive set-up, this means that $\varphi \vee \psi$ enforces the introduction of two extensions of the agent's intentional state. To incorporate these, we have to lift the notion of intentional state. It can no longer be represented by one situation and the expectations our agents have about this situation. It is to be a set of situations and the expectations the agents have about each of these.

\section{Definition 16 (Lifted intentional state)}

Fix $D, A$, and $b$ as before. Define an interpretation $I$ that assigns to every individual constant $c$ an element $I(c) \in D$, and to every noun $N$ and a total function $I(N)$ from $D$ into $\{$ yes, no $\}$.

A state $S$ based on $A, D$ and $I$ is a set of basic states with the following properties:

a. i. For every $\sigma=\left\langle\alpha_{\sigma}, \epsilon_{\sigma}\right\rangle \in S$, it holds that if $c$ is an individual constant, then $I_{\alpha_{\sigma}}(c)=I_{\epsilon_{\sigma}}(c)=I(c)$.

ii. For every $\sigma=\left\langle\alpha_{\sigma}, \epsilon_{\sigma}\right\rangle \in S$, it holds that if $N$ is a noun, then $I_{\alpha_{\sigma}}(N)=$ $I_{\epsilon_{\sigma}}(N)=I(N)$.

iii. $\varnothing$ is the absurd state; the minimal state is the state $\{\mu\}$, where $\mu$ is the minimal basic state.

b. If $\sigma \in S$, then there is no stronger $\sigma^{\prime} \in S$.

Condition (a.) enforces that we restrict ourselves to cases where the addressee is well aware of:

- the set of objects (s)he is talking about - that is why we have the same domain $D$ in all basic states in $S$;

- the names of these objects, and the kind of object they are - that is why the interpretation of the names and the nouns is constant throughout the state.

This way, we can concentrate on the interpretation of the adjectives.

\footnotetext{
43 To deal with this, one has to give a slightly different interpretation to might than the original update definition in Veltman (1996). In this constructive set-up the following works: $S[\operatorname{might} \varphi]=S$ iff there exists a strengthening $S^{\prime}$ of $S$ such that $S^{\prime} \models \varphi$; otherwise, $S[\operatorname{might} \varphi]=\emptyset$.
} 
Condition (b.) makes a state an antichain in the set of the basic states ordered by the stronger-than relation. If a basic state $\sigma \in S$, then no stronger basic state $\sigma^{\prime}$ can be an element of $S$.

Why this condition? We could have taken a different route; we could have stipulated that if $\sigma \in S$, all basic states stronger than $\sigma$ should be elements of $S$ as well. That would have worked, too. But it is all or none. In the all case, $S$ would include for every $\sigma \in S$ the possible ways in which $\sigma$ can develop, while the weakest elements of $S$ would represent the 'actual' information and expectations of the agent. But we take the none option, and choose to put only these weakest elements in the state $S$. After all, with this set the possible ways in which it can develop is implicitly given.

It would be very strange if a state $S$ could contain some but not all extensions of a some basic state $\sigma$. It would only make sense to allow this if for some reason $\sigma$ can only develop into the basic states explicitly incorporated in $S$. But we will encounter no such reason. In our set-up every $\sigma \in S$ can develop into any (coherent) basic state $\sigma^{\prime}$ stronger than $\sigma$.

We also need to lift the stronger-than relation:

Definition 17 Let $S$ and $S^{\prime}$ be states. Then $S^{\prime}$ is at least as strong as $S$ if every $\sigma^{\prime} \in S^{\prime}$ is at least as strong (in the sense of Definition 5) as some $\sigma \in S$.

We have to reformulate the update conditions we have given so far. We do not have to start all over again. Referring to the definitions given so far we can stipulate that for all $\varphi$ dealt with so far the following will hold.

$$
\begin{aligned}
& S \llbracket \varphi, N, C \rrbracket^{+}=\min \left\{\sigma^{\prime} \mid \sigma^{\prime}=\sigma \llbracket G(a), N, C \rrbracket^{+} \text {for some } \sigma \in S\right\} . \\
& S \llbracket \varphi, N, C \rrbracket^{-}=\min \left\{\sigma^{\prime} \mid \sigma^{\prime}=\sigma \llbracket G(a), N, C \rrbracket^{-} \text {for some } \sigma \in S\right\} .
\end{aligned}
$$

The min-operation is enforces that the result of the update with $\varphi$ will satisfy the antichain condition. It is defined as follows.

\section{Definition 18}

Let $\Sigma$ be a set of basic states. Then

$\min \Sigma=\left\{\sigma \in \Sigma \mid\right.$ there is no $\sigma^{\prime} \in \Sigma$ such that $\sigma^{\prime}$ is weaker than $\left.\sigma\right\}$.

\section{Definition 19 (Conjunction and disjunction)}

$S \llbracket \varphi \wedge \psi, N, C \rrbracket^{+}=\min \left\{\sigma^{\prime} \mid \sigma^{\prime}=\sigma \llbracket \varphi, N, C \rrbracket^{+} \llbracket \psi, N, C \rrbracket^{+}\right.$for some $\left.\sigma \in S\right\}$

$S \llbracket \varphi \wedge \psi, N, C \rrbracket^{-}=\min \left(S \llbracket \varphi, N, C \rrbracket^{-} \cup S \llbracket \psi, N, C \rrbracket^{-}\right)$

$S \llbracket \varphi \vee \psi, N, C \rrbracket^{+}=\min \left(S \llbracket \varphi, N, C \rrbracket^{+} \cup S \llbracket \psi, N, C \rrbracket^{+}\right)$

$S \llbracket \varphi \vee \psi, N, C \rrbracket^{-}=\min \left\{\sigma^{\prime} \mid \sigma^{\prime}=\sigma \llbracket \varphi, N, C \rrbracket^{-} \llbracket \psi, N, C \rrbracket^{-}\right.$for some $\left.\sigma \in S\right\}$

It is instructive to check that $S \llbracket G(a) \vee G(b), N, C \rrbracket^{+} \models G(a) \vee G(b)$, and to discover that this would not hold if the states were just sets of basic states rather than antichains.

The definitions above are rather naive. They work well as long as the context remains the same throughout the interpretation process. Consider again the case in which we are discussing the height of Yanjing and Fengkui-whom we know to be adult Chinese men- in a context where the given comparison class is, say, Chinese basketball players. But compare: 

a. Yanjing is tall, but Fengkui is not.
b. Bill is tall, and his six-year-old daughter is too. ${ }^{44}$

Clearly, on the preferred reading of (22-b), each of the conjuncts is interpreted in its default context. How does this come about? We leave it as a challenge to describe the mechanisms at play here, and to develop an elegant formalism capturing these.

The same issue will come up with other logical constants. Compare:
a. Everyone in my team is tall.
b. Everyone in my family is tall. ${ }^{45}$

Clearly, in (23-b) each member of the family is judged by its own age and gender. This is evoked by the collective noun family, the members of which normally belong to clearly distinct generations. When the collective noun does not come with such a varied structure in the collection it introduces, as in the case of (23-a), all members are judged by the criteria supplied by the contextually given comparison class.

People do not often assert a sentence of the form All M are $G$ with $G$ a strongly evaluative adjective. This is because in most cases it is difficult, if not impossible, to satisfy the Experience Principle. You cannot sincerely assert All paintings by Rembrandt are beautiful if you have not seen all of them.

This is one of the reasons why it is interesting to look at sentences starting with a bare plural rather than a universal quantifier-Pies are tasty, instead of All Pies are tasty. Sentences of this form often express defaults, rules with exceptions. When you accept Pies are tasty, you will expect every pie to be tasty. This expectation will not always turn out to be correct, but that does not necessarily mean that you will change your mind. You may consider this bad experience an exception that proves the rule, and still expect the next pie to be tasty. Hence, basically what you have to do when you learn that $M$ 's are $G$ is (a) change $I_{\epsilon_{\sigma}}(G, N, C)$ for each $\sigma \in S$ in such a manner that it assigns yes to each $d \in C$ for which $I(M)(d)=$ yes and $I_{\alpha_{\sigma}}(G, N, C)$ is undefined; (b) adapt the result to the general constraints; and (c) for strong evaluatives, see to it that the Universality and Experience Principles hold, the latter for at least some $M$.

\section{Concluding remarks}

The formal theory developed in the preceding sections can be characterised with two keywords: 'state-based' and 'dynamic'. Let us say a few words about each.

State-based: the central logical notion is not truth in a possible world, but acceptance in an intentional state.

Dynamic: We first defined inductively how a state $S$ changes when it is updated with a sentence $\varphi$, and then we defined acceptance globally: if a state $S$ does not change when it is updated with $\varphi$, then $\varphi$ is accepted in $S$. In a static approach we would first have specified the acceptance conditions inductively, and then have defined the notion of update globally. The dynamic perspective helped a lot in developing the

\footnotetext{
44 The example is taken from Kamp and Sassoon (2017).

45 The example is taken from Kennedy (2007), also cited by Kamp and Sassoon (2017).
} 
theory. Still, one might wonder if, from a purely mathematical point of view, we could have proceeded differently. Are there any obstacles that prevent to go the other way? Well, not really - not at least for the simple language formalised in this paper. It is easy to read off the acceptance (and rejection) conditions of atomic sentences from the update conditions definitions we gave. Negation, conjunction and disjunction follow smoothly. And then one can define the update of a state $S$ with a sentence $\varphi$ as the weakest strengthening of $S$ in which $\varphi$ is accepted. ${ }^{46}$

The real advantage of the dynamic set-up will appear when in the next stage of this project it will be studied how comparison bases and comparison classes change along a discourse. We hinted at the mechanisms at play here, but we did not to describe them formally. To do so, we will have to enrich the language at least with complex predicates of the form $G N$ with $G$ an RGA, and $N$ a noun.

Our main goal in this paper was to describe in a formal system what exactly is the case if one agent accepts a statement of the form $x$ is $G$, for $G$ a predicate of personal taste, and another agent rejects this statement. Such a statement is in some sense 'subjective', but we believe that despite this fact it can give rise to a rational discussion. Sometimes it even happens that a discussion makes people change their mind-or rather, their taste.

We also believe that the theory we sketched gives a better picture of what such a disagreement amounts to than competing theories do. The contextualists, the relativists, the expressivists and the absolutists all give a different analysis of what is going on in evaluative disagreements. They all have trouble explaining how, despite the subjectivity involved, sometimes a sensible discussion is possible.

First of all, both on the contextualist and the relativist account it is difficult to see that there is a disagreement to begin with. On the contextualist account each of the the contestants means something else when she says This is $G$, and on the relativist account each of the contestants is talking about a different world.

More precisely, on the contextualist account This is $G$ expresses a different proposition in different utterance contexts. The utterance context is supposed to provide the standard for $G$, and on most accounts this standard is provided by the speaker. That way This is $G$ becomes equivalent to I find this $G$.

On the relativist account, ${ }^{47}$ This is $G$ does express the same proposition for both contestants, but they are referring to different worlds. Every world comes with some judge who provides the standard for $G$. On most accounts, a speaker asserting This is $G$ is implicitly referring to the world in which she is the judge-as if she is saying: This is $G$ where I come from. ${ }^{48}$

Some relativists, in particular MacFarlane (2007), claim that when a speaker asserts This is $G$ she is implicitly referring to the addressee's world-claiming the addressee's agreement. In our approach, the intuitive idea behind this construction is built into the

\footnotetext{
46 See Yalcin and Rothschild (2017) for an abstract (but detailed) investigation of the conditions under which a dynamic set-up can be replaced by a static setup.

47 This view originated with Lasersohn (2005) as a reaction against contextualism. See Glanzberg (2007) for a critique of the relativist position and a defense of the contextualist point of view.

48 These short characterisations of the various 'schools' of thought concerning predicates of personal taste do not do justice to the many insights built in in these these frameworks. The reader can find more thorough literature reviews in, e.g., Pearson (2013) and Umbach (2016).
} 
very architecture of the system. It is the addressee's state that is being updated, and it is via the addressee's taste, or formally, via the addressee's test function, that the acceptabilty of This is $G$ is determined.

We took much inspiration from the expressivist position taken by Buekens (2011). On his account, an utterance of the form $x$ is $G$, for $G$ a predicate of personal taste, has both an assertive and an expressive dimension. When I say $x$ is $G$, and you say $x$ is not $G$, what is asserted is that we have opposite attitudes towards the object $x$ : I find $x G$, you don't-our tastes differ. However, according to Buekens we do not only assert that we have opposite attitudes, we also express these opposite attitudes. It is at this non-cognitive level that our attitudes clash, that's where the disagreement lies. Unfortunately, at this non-cognitive level, no rational discussion is possible.

According to Buekens, both the contextualists and the relativists neglect the expressive dimension, which is why they have trouble explaining how a taste disagreement can arise. We think our account does better in this respect, with the machinery of test functions and expectations.

Absolutists stand against the relativisation of truth. Diehard absolutists would argue that, contrary to what one may think, there is no subjectivity in adjectives like tasty or beautiful, that these express objective properties. ${ }^{49}$ More nuanced forms, defended in Moltmann (2010) and Pearson (2013), come closer to our position. Both of them maintain, just like we do, that taste judgments of the form This is $G$ make claims beyond the individual speaker. On Pearson's view This is G roughly means People in general find this $G$, for Moltmann the generalisation is more restricted and concerns what she calls "first-person-oriented genericity", which is a form of generalisation that in English is associated with the generic pronoun one: One finds this tasty. Now, on both analyses This is $G$ has absolute truth conditions. And on both accounts it looks like evaluative disagreements can be settled by sociologists. On our account $x$ is $G$ implies People should find this $G$ rather than People do find this $G$, but Absolutists might at least like the universalist stance in our set-up.

Evaluative disagreements are disagreements like any other disagreement. They are just very difficult to resolve. Changing your mind about the tastiness of a certain dish, or the beauty of a certain song, is not just a matter of changing your beliefs. It really involves changing your taste. That is why we built in test functions in our models. Words are not enough to make you like a particular kind of beer, if you did not like it right away. You will have to taste it again, and maybe learn to appreciate its 'delicate bitterness'.

Words are not enough, but they may help. Our taste is influenced by other people's tastes. A review may make us realise that the song we liked so much is rather kitschyand now we cannot listen to it anymore. Or maybe now that you understand it better, you find the poem that you found (intriguing but) difficult to understand when you first read it, really beautiful.

Tastes differ, but they can change, and sometimes words can make them change. It is not for semantics to investigate whether words can make tastes change for the better-cultivate them-but at least it should leave the possibility open.

\footnotetext{
49 Recent support for this view is found in Smith (2007).
} 
Acknowledgements We gratefully acknowledge support from the Netherlands Organisation for Scientific Research (NWO) to the VAAG-Project "Vagueness, approximation and granularity" (231-80-004). This project was a component of the EUROCORES Programme "Modelling Intelligent Interaction-Logic in the Humanities, Social and Computational Sciences" coordinated by the European Science Foundation. The ideas underlying this paper have been presented at various venues: University of Tilburg, University of Utrecht, Peking University, the TARK summerschool at the University of Maryland, University of Barcelona, Rutgers University, Université Paris-Diderot. We are grateful to the audiences for the feedback they provided. We also want to thank Robert van Rooij and Martin Stokhof, whose questions and comments were constitutive for the development of our thoughts. Finally, the feedback of the two anonymous reviewers made us change our mind on a number of issues and forced us to be a lot clearer than we originally were.

Open Access This article is distributed under the terms of the Creative Commons Attribution 4.0 International License (http://creativecommons.org/licenses/by/4.0/), which permits unrestricted use, distribution, and reproduction in any medium, provided you give appropriate credit to the original author(s) and the source, provide a link to the Creative Commons license, and indicate if changes were made.

\section{References}

Asher, N. (2011). Lexical meaning in context: A web of words. Cambridge: Cambridge University Press.

Barker, C. (2002). The dynamics of vagueness. Linguistics and Philosophy, 25(1), 1-36.

Barker, C. (2013). Negotiating taste. Inquiry, 56(2-3), 240-257.

Bartsch, R., \& Venneman, T. (1972). Semantic structures: Semantic structures: A study in the relation between syntax and semantics. Frankfurt: Athenäum Verlag.

Bierwisch, M. (1989). The semantics of gradation. In M. Bierwisch \& E. Lang (Eds.), Dimensional adjectives (pp. 71-261). Berlin: Springer.

Buekens, F. (2011). Faultless disagreement, assertions and the affective-expressive dimension of judgments of taste. Philosophia, 39(4), 637-655.

Burnett, H. S. (2012). The grammar of tolerance on vagueness, context-sensitivity, and the origin of scale structure. Ph.D. thesis, UC Los Angeles.

Crespo, I., Karawani, H., \& Veltman, F. (2018). Expressing expectations. In D. Ball \& B. Rabern (Eds.), The science of meaning (pp. 253-276). Oxford: Oxford University Press.

Crespo, M. I. (2015). Affecting meaning: Subjectivity and evaluativity in gradable adjectives. Ph.D. thesis, ILLC, University of Amsterdam.

Doetjes, J. S., Constantinescu, C., \& Součková, K. (2009). A neo-Kleinian approach to comparatives. Semantics and Linguistic Theory, 19, 124-141.

Dummett, M. (1975). Wang's paradox. Synthese, 30(3-4), 201-32.

Égré, P. (2015). Vagueness: Why do we believe in tolerance? Journal of Philosophical Logic, 44(6), 663679.

Égré, P., \& Cova, F. (2015). Moral asymmetries and the semantics of many. Semantics and Pragmatics, 8, $1-45$.

Fara, D. G. (2000). Shifting sands: An interest relative theory of vagueness. Philosophical Topics, 28(1), 45-81. Originally published under the name "Delia Graff".

Fernando, T., \& Kamp, H. (1996). Expecting many. Semantics and Linguistic Theory, 6, 53-68.

Fine, K. (1975). Vagueness, truth, and logic. Synthese, 30(3-4), 265-300.

Ginsborg, H. (1990). Reflective judgment and taste. Noûs, 24(1), 63-78.

Glanzberg, M. (2007). Context, content, and relativism. Philosophical Studies, 136(1), 1-29.

Groenendijk, J., \& Stokhof, M. (1984). Studies on the semantics of questions and the pragmatics of answers. Ph.D. thesis, University of Amsterdam.

Guyer, P. (1979). Kant and the claims of taste. Cambridge, MA: Harvard University Press.

Kamp, H. (1981). The paradox of the heap. In U. Mönnich (Ed.), Aspects of philosophical logic (pp. 225-277). Berlin: Springer.

Kamp, H., \& Sassoon, G. W. (2017). Vagueness. In P. D. M. Aloni (Ed.), The Cambridge handbook of formal semantics (pp. 389-441). Cambridge, MA: Cambridge University Press.

Kant, I. (1790). Critique of the power of judgment. P. Guyer (Ed.), The Cambridge Edition of the Works of Immanuel Kant, trans. by P. Guyer and E. Matthews. Cambridge: Cambridge University Press (2000). 
Kennedy, C. (2007). Vagueness and grammar: The semantics of relative and absolute gradable adjectives. Linguistics and Philosophy, 30(1), 1-45.

Kennedy, C. (2013). Two sources of subjectivity: Qualitative assessment and dimensional uncertainty. Inquiry, 56(2-3), 258-277.

Kennedy, C., \& McNally, L. (2005). Scale structure, degree modification, and the semantics of gradable predicates. Language, 81(2), 345-79.

Lasersohn, P. (2005). Context dependence, disagreement, and predicates of personal taste. Linguistics and Philosophy, 28(6), 643-686.

MacFarlane, J. (2007). Relativism and disagreement. Philosophical Studies, 132(1), 17-31.

MacFarlane, J. (2014). Assessment sensitivity-Relative truth and its applications. Oxford: Oxford University Press.

Moltmann, F. (2010). Relative truth and the first person. Philosophical Studies, 150(2), 187-220.

Morzycki, M. (2015). Modification. Cambridge: Cambridge University Press.

Ninan, D. (2014). Taste predicates and the acquaintance inference. Semantics and Linguistic Theory, 24, 290-309.

Pearson, H. (2013). A judge-free semantics for predicates of personal taste. Journal of Semantics, 30(1), $103-154$.

Sæbø, K. J. (2009). Judgment ascriptions. Linguistics and Philosophy, 32(4), 327-352.

Sassoon G.W. (2010). Restricted quantification over tastes. In M. Aloni, H. Bastiaanse, T. de Jager, \& K. Schulz (Eds.), Logic, language and meaning. 17th Amsterdam Colloquium. Revised selected papers (pp. 163-172). Berlin: Springer.

Sassoon, G. W. (2013). A typology of multidimensional adjectives. Journal of Semantics, 30(3), 335-380.

Siegel, M. (1976). Capturing the Adjective. Ph.D. thesis, University of Massachusetts at Amherst.

Smith, B. C. (2007). The objectivity of taste and tasting. In B. C. Smith (Ed.), Questions of taste: The philosophy of wine (pp. 41-76). Oxford: Oxford University Press.

Stephenson, T. (2006). Towards a theory of subjective meaning. Ph.D. thesis, MIT.

Stephenson, T. (2007). Judge dependence, epistemic modals, and predicates of personal taste. Linguistics and Philosophy, 30(4), 487-525.

Umbach, C. (2016). Evaluative propositions and subjective judgments. In C. Meier \& J. van WijnbergenHuitink (Eds.), Subjective meaning: Alternatives to relativism (pp. 127-168). Berlin: De Gruyter.

Veltman, F. (1996). Defaults in update semantics. Journal of Philosophical Logic, 25(3), 221-261.

Weiss, S. E. (1976). The sorites fallacy: What difference does a peanut make? Synthese, 33(2-4), 253-272.

Wittgenstein, L. (1958). Philosophical investigations (2nd ed.). Oxford: Blackwell.

Yalcin, S., \& Rothschild, D. (2017). On the dynamics of conversation. Noûs, 51(1), 24-48.

Publisher's Note Springer Nature remains neutral with regard to jurisdictional claims in published maps and institutional affiliations. 